\title{
Linear Hyperbolic Functional-Differential Equations with Essentially Bounded Right-Hand Side
}

\author{
Alexander Domoshnitsky, ${ }^{1}$ Alexander Lomtatidze, ${ }^{2}$ \\ Abraham Maghakyan, ${ }^{1}$ and Jiři Šremr ${ }^{2}$ \\ ${ }^{1}$ Department of Mathematics and Computer Science, Ariel University Center of Samaria, \\ 40700 Ariel, Israel \\ ${ }^{2}$ Institute of Mathematics, Academy of Sciences of the Czech Republic, Branch in Brno, Žižkova 22, \\ 61662 Brno, Czech Republic
}

Correspondence should be addressed to Alexander Domoshnitsky, adom@ariel.ac.il

Received 14 March 2011; Revised 18 May 2011; Accepted 19 May 2011

Academic Editor: Stefan Siegmund

Copyright (C) 2011 Alexander Domoshnitsky et al. This is an open access article distributed under the Creative Commons Attribution License, which permits unrestricted use, distribution, and reproduction in any medium, provided the original work is properly cited.

Theorems on the unique solvability and nonnegativity of solutions to the characteristic initial value problem $u^{(1,1)}(t, x)=\ell_{0}(u)(t, x)+\ell_{1}\left(u^{(1,0)}\right)(t, x)+\ell_{2}\left(u^{(0,1)}\right)(t, x)+q(t, x), u(t, c)=\alpha(t)$ for $t \in$ $[a, b], u(a, x)=\beta(x)$ for $x \in[c, d]$ given on the rectangle $[a, b] \times[c, d]$ are established, where the linear operators $\ell_{0}, \ell_{1}, \ell_{2}$ map suitable function spaces into the space of essentially bounded functions. General results are applied to the hyperbolic equations with essentially bounded coefficients and argument deviations.

\section{Introduction}

On the rectangle $\Phi=[a, b] \times[c, d]$, we consider the linear partial functional-differential equation

$$
u^{(1,1)}(t, x)=\ell_{0}(u)(t, x)+\ell_{1}\left(u^{(1,0)}\right)(t, x)+\ell_{2}\left(u^{(0,1)}\right)(t, x)+q(t, x)
$$

where $u^{(1,0)}$ and $u^{(0,1)}$ (resp., $\left.u^{(1,1)}\right)$ denote the first-order (resp., the second-order mixed) partial derivatives. The operators $\ell_{0}, \ell_{1}$, and $\ell_{2}$ are supposed to be linear and acting from suitable function spaces (see Section 3 ) to the space of Lebesgue measurable and essentially bounded functions. By a solution to (1.1), we mean a function $u: \Phi \rightarrow \mathbb{R}$ absolutely 
continuous in the sense of Carathéodory possessing some additional properties (namely, inclusions (2.20)) which satisfies equality (1.1) almost everywhere on $\boldsymbol{\Phi}$.

Three main initial value problems for the hyperbolic equations are studied in the literature-Darboux, Cauchy, and Goursat problems. In this paper, we consider the Darboux problem in which case the values of a solution $u$ to (1.1) are prescribed on both characteristics $t=a$ and $x=c$, that is, the initial conditions are

$$
u(t, c)=\alpha(t) \quad \text { for } t \in[a, b], \quad u(a, x)=\beta(x) \quad \text { for } x \in[c, d]
$$

Properties of the initial functions $\alpha$ and $\beta$ will be specified in Section 3. It is worth to remember here that various initial and boundary value problems for the hyperbolic equation

$$
u_{t x}=f\left(t, x, u, u_{t}, u_{x}\right)
$$

with continuous as well as discontinuous right-hand sides but without argument deviations have been studied in detail (see, e.g., [1-13] and references therein). As for the hyperbolic functional-differential equations, we can mention for example the works [14-16] (see also references cited therein) but, as far as the authors know, there is still a broad field for further investigation. We have made the first steps in the papers $[17,18]$ where the Darboux problem for (1.1) with $\ell_{1}=0$ and $\ell_{2}=0$ is considered.

\section{Notation and Definitions}

The following notation is used throughout the paper.

(i) $\mathbb{N}, \mathbb{Q}$, and $\mathbb{R}$ are the sets of all natural, rational, and real numbers, respectively, $\mathbb{R}_{+}=$ $[0,+\infty[$.

(ii) $\Phi=[a, b] \times[c, d]$, where $-\infty<a<b<+\infty$ and $-\infty<c<d<+\infty$.

(iii) The first-order partial derivatives of the function $u: \Phi \rightarrow \mathbb{R}$ at the point $(t, x) \in \Phi$ are denoted by $u^{(1,0)}(t, x)$ (or $u_{t}(t, x)$ ) and $u^{(0,1)}(t, x)$ (or $u_{x}(t, x)$ ). The second-order mixed partial derivatives of the function $u: \Phi \rightarrow \mathbb{R}$ at the point $(t, x) \in \Phi$ are denoted by $u_{t x}(t, x)$ and $u_{x t}(t, x)$ whereas we use $u^{(1,1)}(t, x)$ if $u_{t x}(t, x)=u_{x t}(t, x)$.

(iv) $C(\Phi ; \mathbb{R})$ is the Banach space of continuous functions $u: \Phi \rightarrow \mathbb{R}$ equipped with the norm

$$
\|u\|_{C}=\max \{|u(t, x)|:(t, x) \in \Phi\} .
$$

(v) $C([\alpha, \beta] ; \mathbb{R})$, where $-\infty<\alpha<\beta<+\infty$, is the linear space of continuous functions $v:[\alpha, \beta] \rightarrow \mathbb{R}$.

(vi) $A C([\alpha, \beta] ; \mathbb{R})$, where $-\infty<\alpha<\beta<+\infty$, is the linear space of absolutely continuous functions $v:[\alpha, \beta] \rightarrow \mathbb{R}$. 
(vii) $L^{\infty}(\Phi ; \mathbb{R})$ is the Banach space of Lebesgue measurable and essentially bounded functions $p: \Phi \rightarrow \mathbb{R}$ equipped with the norm

$$
\|p\|_{L^{\infty}}=\operatorname{ess} \sup \{|p(t, x)|:(t, x) \in \boldsymbol{\Phi}\} .
$$

(viii) $L^{\infty}\left(\boldsymbol{\Phi} ; \mathbb{R}_{+}\right)=\left\{p \in L^{\infty}(\boldsymbol{\Phi} ; \mathbb{R}): p(t, x) \geq 0\right.$ for a.e. $\left.(t, x) \in \boldsymbol{\Phi}\right\}$.

(ix) For any $z_{1}, z_{2} \in L^{\infty}(\Phi ; \mathbb{R})$, we put

$$
\begin{gathered}
z_{2} \geq z_{1} \Longleftrightarrow z_{2}(t, x)-z_{1}(t, x) \geq 0 \quad \text { for a.e. }(t, x) \in \Phi \\
z_{2} \gg z_{1} \Longleftrightarrow z_{2}(t, x)-z_{1}(t, x) \geq \varepsilon \quad \text { for a.e. }(t, x) \in \Phi \text { with some } \varepsilon>0 .
\end{gathered}
$$

(x) $L^{\infty}([\alpha, \beta] ; \mathbb{R})$, where $-\infty<\alpha<\beta<+\infty$, is the linear space of Lebesgue measurable and essentially bounded functions $f:[\alpha, \beta] \rightarrow \mathbb{R}$.

(xi) meas $A$ denotes the Lebesgue measure of the set $A \subset R^{m}, m=1,2$.

(xii) If $X, Y$ are Banach spaces and $T: X \rightarrow Y$ is a linear bounded operator then $\|T\|$ denotes the norm of the operator $T$, that is,

$$
\|T\|=\sup \left\{\|T(z)\|_{Y}: z \in X,\|z\|_{X} \leq 1\right\} .
$$

Two subsections below contain a number of definitions used in the sequel.

\subsection{Spaces $Z_{[1]}(\Phi ; \mathbb{R}), Z_{[2]}(\Phi ; \mathbb{R})$, and $\operatorname{Set} C^{*}(\Phi ; \mathbb{R})$}

Motivated by [19, Section 2], the authors introduce the following assertions and definitions.

Lemma 2.1 (see $[19$, Section 1, Lemma 1]). Let the function $u: \Phi \rightarrow \mathbb{R}$ be such that

$$
\begin{gathered}
u(\cdot, x):[a, b] \longrightarrow \mathbb{R} \text { is continuous for a.e. } x \in[c, d], \\
u(t, \cdot):[c, d] \longrightarrow \mathbb{R} \text { is measurable for all } t \in[a, b] .
\end{gathered}
$$

Then the function $\max \{|u(t, \cdot)|: t \in[a, b]\}:[c, d] \rightarrow \mathbb{R}$ is measurable.

Notation 1. $Z_{[1]}(\Phi ; \mathbb{R})$ denotes the linear space of all functions $u: \Phi \rightarrow \mathbb{R}$ satisfying conditions (2.5), and

$$
\text { ess } \sup \{\max \{|u(t, x)|: t \in[a, b]\}: x \in[c, d]\}<+\infty \text {. }
$$


If one identifies functions $u_{1}, u_{2}$ from $Z_{[1]}(\Phi ; \mathbb{R})$ such that $u_{1}(\cdot, x) \equiv u_{2}(\cdot, x)$ for a.e. $x \in[c, d]$ then

$$
\|u\|_{Z_{[1]}}=\operatorname{ess} \sup \{\max \{|u(t, x)|: t \in[a, b]\}: x \in[c, d]\}
$$

defines a norm in the space $Z_{[1]}(\Phi ; \mathbb{R})$.

Analogously, we introduce the space $Z_{[2]}(\boldsymbol{\Phi} ; \mathbb{R})$ of functions which are "measurable in the first variable and continuous in the second one" and define the norm $\|\cdot\|_{Z_{[2]}}$ there.

The proof of the following proposition is similar to those presented in [19, Section 2, Lemma 1]. For the sake of completeness we prove the proposition here in detail.

Proposition 2.2. $Z_{[1]}(\boldsymbol{\Phi} ; \mathbb{R})$ and $Z_{[2]}(\Phi ; \mathbb{R})$ are Banach spaces.

Proof. We only prove the assertion for the space $Z_{[1]}(\boldsymbol{\Phi} ; \mathbb{R})$, the assertion of the lemma concerning the space $Z_{[2]}(\Phi ; \mathbb{R})$ can be proven analogously by exchanging the roles of the variables $t$ and $x$.

Let $\left\{u_{k}\right\}_{k=1}^{+\infty}$ be an arbitrary Cauchy sequence in $Z_{[1]}(\Phi ; \mathbb{R})$. For a decreasing sequence of positive numbers $\left\{\varepsilon_{i}\right\}_{i=1}^{+\infty}$ with $\sum_{i=1}^{+\infty} \varepsilon_{i}<+\infty$ there exists an increasing sequence $\left\{k_{i}\right\}_{i=1}^{+\infty}$ such that

$$
\text { ess } \sup \left\{\max \left\{\left|u_{n}(t, x)-u_{k}(t, x)\right|: t \in[a, b]\right\}: x \in[c, d]\right\}<\varepsilon_{i}
$$

for every $n, k \geq k_{i}, i \in \mathbb{N}$. Let $v_{i}=u_{k_{i}}(i=1,2, \ldots)$. Then, for any $i \in \mathbb{N}$, there is a set $E_{i} \subseteq[c, d]$, meas $E_{i}=d-c$, such that

$$
\max \left\{\left|v_{i+1}(t, x)-v_{i}(t, x)\right|: t \in[a, b]\right\}<\varepsilon_{i} \quad \text { for } x \in E_{i}, i \in \mathbb{N}
$$

Put $E=\cap_{i=1}^{+\infty} E_{i}$. Then, clearly, we have meas $E=d-c$ and

$$
\begin{aligned}
\max & \left\{\left|v_{n}(t, x)-v_{k}(t, x)\right|: t \in[a, b]\right\} \\
& \leq \sum_{m=k}^{n-1} \max \left\{\left|v_{m+1}(t, x)-v_{m}(t, x)\right|: t \in[a, b]\right\} \\
& \leq \sum_{m=k}^{+\infty} \varepsilon_{m} \text { for } x \in E, n>k .
\end{aligned}
$$

Consequently, for any fixed $x \in E$, the sequence $\left\{v_{i}(\cdot, x)\right\}_{i=1}^{+\infty}$ converges uniformly on $[a, b]$, say to $u(\cdot, x)$. Hence, $\left\{v_{i}(t, \cdot)\right\}_{i=1}^{+\infty}$ converges point-wise on $E$ to $u(t, \cdot)$ for every fixed $t \in[a, b]$. Therefore, the function $u$ satisfies conditions (2.5). Since

$$
\begin{aligned}
u_{k}(t, x)-u(t, x) & =u_{k}(t, x)-u_{k_{i}}(t, x)+\lim _{n \rightarrow+\infty}\left[v_{i}(t, x)-v_{n}(t, x)\right] \\
& =u_{k}(t, x)-u_{k_{i}}(t, x)+\lim _{n \rightarrow+\infty} \sum_{m=i}^{n-1}\left[v_{m}(t, x)-v_{m+1}(t, x)\right]
\end{aligned}
$$


holds for $i, k \in \mathbb{N}$, all $t \in[a, b]$ and a.e. $x \in[c, d]$, in view of (2.8) and (2.9), we obtain

$$
\left\|u_{k}-u\right\|_{Z_{[1]}} \leq \varepsilon_{i}+\sum_{m=i}^{+\infty} \varepsilon_{m} \text { for } k \geq k_{i}, i \in \mathbb{N}
$$

Hence, $u \in Z_{[1]}(\boldsymbol{\Phi} ; \mathbb{R})$ and $u_{n} \rightarrow u$ in $Z_{[1]}(\boldsymbol{\Phi} ; \mathbb{R})$, that is, the space $Z_{[1]}(\boldsymbol{\Phi} ; \mathbb{R})$ is complete.

For the investigation of hyperbolic differential equations with discontinuous righthand side, the concept of a Carathéodory solution is usually used (see, e.g., $[7,10,20,21]$ ), that is, solutions are considered in the class of absolutely continuous functions. One possible definition of absolute continuity of functions of two variables was given by Carathéodory in his monograph [22]. It is also known that such functions admit a certain integral representation. Following the concept mentioned, we introduce the following.

Notation 2. $C^{*}(\Phi ; \mathbb{R})$ stands for the set of functions $u: \Phi \rightarrow \mathbb{R}$ admitting the integral representation

$$
u(t, x)=z+\int_{a}^{t} f(s) \mathrm{d} s+\int_{c}^{x} g(\eta) \mathrm{d} \eta+\int_{a}^{t} \int_{c}^{x} h(s, \eta) \mathrm{d} \eta \mathrm{d} s \quad \text { for }(t, x) \in \Phi,
$$

where $z \in \mathbb{R}, f \in L^{\infty}([a, b] ; \mathbb{R}), g \in L^{\infty}([c, d] ; \mathbb{R})$, and $h \in L^{\infty}(\boldsymbol{\Phi} ; \mathbb{R})$.

The next lemma on differentiating of an indefinite double integral plays a crucial role in our investigation.

Lemma 2.3 (see [23, Proposition 3.5]). Let $h: \Phi \rightarrow \mathbb{R}$ be a Lebesgue integrable function and

$$
v(t, x)=\int_{a}^{t} \int_{c}^{x} h(s, \eta) \mathrm{d} \eta \mathrm{d} s \quad \text { for }(t, x) \in \Phi
$$

Then

(1) there exists a set $E \subseteq[a, b]$ such that meas $E=b-a$ and

$$
v^{(1,0)}(t, x)=\int_{c}^{x} h(t, \eta) \mathrm{d} \eta \quad \text { for } t \in E, x \in[c, d]
$$

(2) there exists a set $F \subseteq[c, d]$ such that meas $F=d-c$ and

$$
v^{(0,1)}(t, x)=\int_{a}^{t} h(s, x) \mathrm{d} s \quad \text { for } t \in[a, b] \text { and } x \in F,
$$

(3) there exists a set $G \subseteq \Phi$ such that meas $G=(b-a)(d-c)$ and

$$
v^{(1,1)}(t, x)=h(t, x) \quad \text { for }(t, x) \in G \text {. }
$$


Remark 2.4. If $u \in C^{*}(\Phi ; \mathbb{R})$, that is, the function $u$ admits integral representation (2.13), then by using Lemma 2.3 we get

$$
\begin{aligned}
& u^{(1,0)}(t, x)=f(t)+\int_{c}^{x} h(t, \eta) \mathrm{d} \eta \quad \text { for a.e. } t \in[a, b] \text { and all } x \in[c, d] \\
& u^{(0,1)}(t, x)=g(x)+\int_{a}^{t} h(s, x) \mathrm{d} s \quad \text { for all } t \in[a, b] \text { and a.e. } x \in[c, d] \\
& u^{(1,1)}(t, x)=h(t, x) \quad \text { for a.e. }(t, x) \in \boldsymbol{\Phi} .
\end{aligned}
$$

Consequently, for any $u \in C^{*}(\Phi ; \mathbb{R})$, we have

$$
u^{(1,0)} \in Z_{[2]}(\boldsymbol{\Phi} ; \mathbb{R}), \quad u^{(0,1)} \in Z_{[1]}(\boldsymbol{\Phi} ; \mathbb{R}), \quad u^{(1,1)} \in L^{\infty}(\boldsymbol{\Phi} ; \mathbb{R})
$$

Remark 2.5. It follows from Remark 2.4 and [22, Satz 1 , page 654] that $u \in C^{*}(\Phi ; \mathbb{R})$ if and only if $u: \Phi \rightarrow \mathbb{R}$ is absolutely continuous in the sense of Carathéodory with the properties

$$
u^{(1,0)}(\cdot, c) \in L^{\infty}([a, b] ; \mathbb{R}), \quad u^{(0,1)}(a, \cdot) \in L^{\infty}([c, d] ; \mathbb{R}), \quad u^{(1,1)} \in L^{\infty}(\Phi ; \mathbb{R})
$$

\subsection{Positive and Volterra-Type Operators}

We recall here some definitions from the theory of linear operators. We start with the operators acting on the space $C(\Phi ; \mathbb{R})$.

Definition 2.6. A linear operator $\ell: C(\boldsymbol{\Phi} ; \mathbb{R}) \rightarrow L^{\infty}(\boldsymbol{\Phi} ; \mathbb{R})$ is said to be positive if the relation

$$
\ell(u)(t, x) \geq 0 \text { for a.e. }(t, x) \in \mathcal{D}
$$

holds whenever the function $u \in C(\Phi ; \mathbb{R})$ is such that

$$
u(t, x) \geq 0 \quad \text { for }(t, x) \in \Phi
$$

Example 2.7. For any $v \in C(\boldsymbol{\Phi} ; \mathbb{R})$, we put

$$
\ell_{0}(v)(t, x)=p_{0}(t, x) v\left(\tau_{0}(t, x), \mu_{0}(t, x)\right) \quad \text { for a.e. }(t, x) \in \Phi
$$

where $p_{0} \in L^{\infty}(\Phi ; \mathbb{R})$ and $\tau_{0}: \Phi \rightarrow[a, b], \mu_{0}: \Phi \rightarrow[c, d]$ are measurable functions. Then the operator $\ell_{0}: C(\boldsymbol{\Phi} ; \mathbb{R}) \rightarrow L^{\infty}(\boldsymbol{\Phi} ; \mathbb{R})$ is linear and bounded. Moreover, $\ell_{0}$ is positive if and only if $p_{0}(t, x) \geq 0$ for a.e. $(t, x) \in \Phi$. 
Definition 2.8. A linear operator $\ell: C(\Phi ; \mathbb{R}) \rightarrow L^{\infty}(\boldsymbol{\Phi} ; \mathbb{R})$ is called $(a, c)$-Volterra operator if, for any $\left(t_{0}, x_{0}\right) \in \Phi$ and $u \in C(\Phi ; \mathbb{R})$ such that

$$
u(t, x)=0 \quad \text { for }(t, x) \in\left[a, t_{0}\right] \times\left[c, x_{0}\right]
$$

we have

$$
\ell(u)(t, x)=0 \text { for a.e. }(t, x) \in\left[a, t_{0}\right] \times\left[c, x_{0}\right]
$$

Remark 2.9. It can be shown by using Lemma 5.8 stated below that the operator $\ell_{0}$ given by formula (2.23) is an $(a, c)$-Volterra one if and only if

$$
\begin{gathered}
\left|p_{0}(t, x)\right|\left(\tau_{0}(t, x)-t\right) \leq 0 \quad \text { for a.e. }(t, x) \in \boldsymbol{\Phi} \\
\left|p_{0}(t, x)\right|\left(\mu_{0}(t, x)-x\right) \leq 0 \quad \text { for a.e. }(t, x) \in \boldsymbol{\Phi}
\end{gathered}
$$

Now we introduce analogous notions for linear operators defined on the spaces $Z_{[1]}(\Phi ; \mathbb{R})$ and $Z_{[2]}(\Phi ; \mathbb{R})$.

Definition 2.10. We say that a linear operator $\ell: Z_{[1]}(\boldsymbol{\Phi} ; \mathbb{R}) \rightarrow L^{\infty}(\boldsymbol{\Phi} ; \mathbb{R})$ (resp., $\ell$ : $\left.Z_{[2]}(\Phi ; \mathbb{R}) \rightarrow L^{\infty}(\Phi ; \mathbb{R})\right)$ is positive if relation $(2.21)$ is satisfied for every function $u \in$ $Z_{[1]}(\Phi ; \mathbb{R})$ (resp., $u \in Z_{[2]}(\Phi ; \mathbb{R})$ ) such that

$$
\begin{aligned}
& u(t, x) \geq 0 \text { for } t \in[a, b] \text { and a.e. } x \in[c, d] \\
& \text { (resp., } u(t, x) \geq 0 \text { for a.e. } t \in[a, b] \text { and all } x \in[c, d]) .
\end{aligned}
$$

Example 2.11. For any $v \in Z_{[2]}(\boldsymbol{\Phi} ; \mathbb{R})\left(\operatorname{resp} ., v \in Z_{[1]}(\boldsymbol{\Phi} ; \mathbb{R})\right)$, we put

$$
\ell_{1}(v)(t, x)=p_{1}(t, x) v\left(t, \mu_{1}(t, x)\right) \text { for a.e. }(t, x) \in \Phi,
$$

respectively,

$$
\ell_{2}(v)(t, x)=p_{2}(t, x) v\left(\tau_{2}(t, x), x\right) \text { for a.e. }(t, x) \in \Phi
$$

where $p_{1}, p_{2} \in L^{\infty}(\boldsymbol{\Phi} ; \mathbb{R})$ and $\mu_{1}: \Phi \rightarrow[c, d], \tau_{2}: \Phi \rightarrow[a, b]$ are measurable functions. Then the operators $\ell_{1}: Z_{[2]}(\boldsymbol{\Phi} ; \mathbb{R}) \rightarrow L^{\infty}(\boldsymbol{\Phi} ; \mathbb{R})$ and $\ell_{2}: Z_{[1]}(\boldsymbol{\Phi} ; \mathbb{R}) \rightarrow L^{\infty}(\boldsymbol{\Phi} ; \mathbb{R})$ are linear and bounded. Moreover, $\ell_{1}$ (resp., $\ell_{2}$ ) is positive if and only if $p_{1}(t, x) \geq 0$ (resp., $p_{2}(t, x) \geq 0$ ) for a.e. $(t, x) \in \Phi$.

Definition 2.12. We say that a linear operator $\ell: Z_{[1]}(\boldsymbol{\Phi} ; \mathbb{R}) \rightarrow L^{\infty}(\boldsymbol{\Phi} ; \mathbb{R})$ (resp., $\ell$ : $Z_{[2]}(\boldsymbol{\Phi} ; \mathbb{R}) \rightarrow L^{\infty}(\boldsymbol{\Phi} ; \mathbb{R})$ ) is an a-Volterra operator (resp., a $c$-Volterra operator) if, for any $t_{0} \in[a, b]$ (resp., $x_{0} \in[c, d]$ ) and $u \in Z_{[1]}(\boldsymbol{\Phi} ; \mathbb{R})$ (resp., $u \in Z_{[2]}(\boldsymbol{\Phi} ; \mathbb{R})$ ) such that

$$
\begin{aligned}
& u(t, x)=0 \text { for } t \in\left[a, t_{0}\right] \text { and a.e. } x \in[c, d] \\
& \text { (resp., } \left.u(t, x)=0 \text { for a.e. } t \in[a, b] \text { and all } x \in\left[c, x_{0}\right]\right),
\end{aligned}
$$


we have

$$
\begin{aligned}
& \ell(u)(t, x)=0 \text { for a.e. }(t, x) \in\left[a, t_{0}\right] \times[c, d] \\
& \left(\text { resp., } \ell(u)(t, x)=0 \text { for a.e. }(t, x) \in[a, b] \times\left[c, x_{0}\right]\right) .
\end{aligned}
$$

Remark 2.13. One can show by using Lemma 5.9 (resp., Lemma 5.10) stated below that the operator $\ell_{1}$ (resp., $\ell_{2}$ ) given by formula (2.28) (resp., (2.29)) is a $c$-Volterra one (resp., an $a$-Volterra one) if and only if

$$
\left|p_{1}(t, x)\right|\left(\mu_{1}(t, x)-x\right) \leq 0 \text { for a.e. }(t, x) \in \boldsymbol{\Phi}
$$

respectively,

$$
\left|p_{2}(t, x)\right|\left(\tau_{2}(t, x)-t\right) \leq 0 \text { for a.e. }(t, x) \in \Phi \text {. }
$$

\section{Statement of Problem}

On the rectangle $\Phi$, we consider the linear nonhomogeneous Darboux problem (1.1), (1.2) in which $\ell_{0}: C(\boldsymbol{\Phi} ; \mathbb{R}) \rightarrow L^{\infty}(\boldsymbol{\Phi} ; \mathbb{R}), \ell_{1}: Z_{[2]}(\boldsymbol{\Phi} ; \mathbb{R}) \rightarrow L^{\infty}(\boldsymbol{\Phi} ; \mathbb{R})$, and $\ell_{2}: Z_{[1]}(\boldsymbol{\Phi} ; \mathbb{R}) \rightarrow L^{\infty}(\boldsymbol{\Phi} ; \mathbb{R})$ are linear bounded operators, $q \in L^{\infty}(\boldsymbol{\Phi} ; \mathbb{R})$, and $\alpha \in A C([a, b] ; \mathbb{R}), \beta \in A C([c, d] ; \mathbb{R})$ are such that $\alpha^{\prime} \in L^{\infty}([a, b] ; \mathbb{R}), \beta^{\prime} \in L^{\infty}([c, d] ; \mathbb{R})$, and $\alpha(a)=\beta(c)$. By a solution to problem (1.1), (1.2), we mean a function $u \in C^{*}(\Phi ; \mathbb{R})$ possessing property (1.2) and satisfying equality (1.1) almost everywhere on $\Phi$. Let us mention that, in view of Remark 2.4, the definition of a solution to the problem considered is meaningful.

We are interested in question on the unique solvability of problem (1.1), (1.2), and nonnegativity of its solutions. Clearly, the second-order hyperbolic differential equation

$$
u_{t x}=p_{0}(t, x) u+p_{1}(t, x) u_{t}+p_{2}(t, x) u_{x}+q(t, x)
$$

where $p_{0}, p_{1}, p_{2}, q \in L^{\infty}(\boldsymbol{\Phi} ; \mathbb{R})$, is a particular case of (1.1). It follows from the results due to Deimling (see [20,21]) that, among others, problem (3.1), (1.2) has a unique solution without any additional assumptions imposed on the coefficients $p_{0}, p_{1}$, and $p_{2}$. We would like to get solvability conditions for general problem (1.1), (1.2) which conform to those well known for (3.1), (1.2).

The main results (namely, Theorems 4.1 and 4.4) will be illustrated on the hyperbolic differential equation with argument deviations

$$
\begin{aligned}
u^{(1,1)}(t, x)= & p_{0}(t, x) u\left(\tau_{0}(t, x), \mu_{0}(t, x)\right)+p_{1}(t, x) u^{(1,0)}\left(t, \mu_{1}(t, x)\right) \\
& +p_{2}(t, x) u^{(0,1)}\left(\tau_{2}(t, x), x\right)+q(t, x)
\end{aligned}
$$

in which coefficients $p_{0}, p_{1}, p_{2}, q \in L^{\infty}(\Phi ; \mathbb{R})$ and argument deviations $\tau_{0}, \tau_{2}: \Phi \rightarrow[a, b]$, $\mu_{0}, \mu_{1}: \nexists \rightarrow[c, d]$ are measurable functions. We obtain this equation from (1.1) if 
the operators $\ell_{0}, \ell_{1}$, and $\ell_{2}$ are defined by formulas (2.23), (2.28), and (2.29), respectively. Let us also mention that in the case, where

$$
\tau_{k}(t, x)=t, \quad \mu_{j}(t, x)=x \quad \text { for a.e. }(t, x) \in \Phi(k=0,2, j=0,1)
$$

equation (3.2) takes form (3.1).

\section{Main Results}

At first, we put

$$
A_{k}(z)=\ell_{k}\left(\varphi_{k}(z)\right) \quad \text { for } z \in L^{\infty}(\Phi ; \mathbb{R}), k=0,1,2
$$

where

$$
\begin{gathered}
\varphi_{0}(z)(t, x)=\int_{a}^{t} \int_{c}^{x} z(s, \eta) \mathrm{d} \eta \mathrm{d} s \quad \text { for }(t, x) \in \Phi, \\
\varphi_{1}(z)(t, x)=\int_{c}^{x} z(t, \eta) \mathrm{d} \eta \quad \text { for a.e. } t \in[a, b] \text { and all } x \in[c, d], \\
\varphi_{2}(z)(t, x)=\int_{a}^{t} z(s, x) \mathrm{d} s \quad \text { for } t \in[a, b], \text { a.e. } x \in[c, d] .
\end{gathered}
$$

Clearly, $\varphi_{0}: L^{\infty}(\Phi ; \mathbb{R}) \rightarrow C(\Phi ; \mathbb{R}), \varphi_{1}: L^{\infty}(\Phi ; \mathbb{R}) \rightarrow Z_{[2]}(\Phi ; \mathbb{R}), \varphi_{2}: L^{\infty}(\boldsymbol{\Phi} ; \mathbb{R}) \rightarrow$ $Z_{[1]}(\boldsymbol{\Phi} ; \mathbb{R})$ and thus the operators $A_{0}, A_{1}, A_{2}$ mapping the space $L^{\infty}(\boldsymbol{\Phi} ; \mathbb{R})$ into itself are linear and bounded.

Theorem 4.1. Let $A=A_{0}+A_{1}+A_{2}$, where the operators $A_{0}, A_{1}, A_{2}$ are defined by relations (4.1), (4.2). If the spectral radius of the operator $A$ is less than one then problem (1.1), (1.2) is uniquely solvable for arbitrary $q \in L^{\infty}(\Phi ; \mathbb{R})$ and $\alpha \in A C([a, b] ; \mathbb{R}), \beta \in A C([c, d] ; \mathbb{R})$ such that $\alpha^{\prime} \in$ $L^{\infty}([a, b] ; \mathbb{R}), \beta^{\prime} \in L^{\infty}([c, d] ; \mathbb{R})$, and $\alpha(a)=\beta(c)$.

Theorem 4.1 implies the following.

Corollary 4.2. If the inequality

$$
(b-a)(d-c)\left\|\ell_{0}\right\|+(d-c)\left\|\ell_{1}\right\|+(b-a)\left\|\ell_{2}\right\|<1
$$

holds then problem (1.1), (1.2) is uniquely solvable for arbitrary $q \in L^{\infty}(\boldsymbol{\Phi} ; \mathbb{R})$ and $\alpha \in$ $A C([a, b] ; \mathbb{R}), \beta \in A C([c, d] ; \mathbb{R})$ such that $\alpha^{\prime} \in L^{\infty}([a, b] ; \mathbb{R}), \beta^{\prime} \in L^{\infty}([c, d] ; \mathbb{R})$, and $\alpha(a)=\beta(c)$.

Remark 4.3. On the rectangle $[a, b] \times[c, d]$, we consider the equation

$$
u^{(1,1)}(t, x)=p_{0} u(b, d)+p_{1} u^{(1,0)}(t, d)+p_{2} u^{(0,1)}(b, x)
$$


subjected to the initial conditions

$$
u(t, c)=0 \quad \text { for } t \in[a, b], \quad u(a, x)=0 \quad \text { for } x \in[c, d],
$$

where

$$
p_{0}=\frac{m_{0}}{(b-a)(d-c)}, \quad p_{1}=\frac{m_{1}}{d-c}, \quad p_{2}=\frac{m_{2}}{b-a} .
$$

Clearly (4.4) is a particular case of (1.1). If $m_{0}+m_{1}+m_{2}=1$, then problem (4.4), (4.5) has the trivial solution $u(t, x) \equiv 0$ and the nontrivial solution $u(t, x) \equiv(t-a)(x-c)$. It justifies that the strict inequality (4.3) in the previous corollary is essential and cannot be replaced by the nonstrict one. On the other hand, it is worth to mention that the inequality indicated is very restrictive and thus it is far from being optimal for a wide class of equations (1.1).

If the operators $\ell_{0}, \ell_{1}$, and $\ell_{2}$ on the right-hand side of (1.1) are positive then we can estimate the spectral radius of the operator $A$ by using the well-known results due to Krasnosel'skij and we thus obtain the following.

Theorem 4.4. Let the operators $\ell_{0}, \ell_{1}, \ell_{2}$ be positive and $A=A_{0}+A_{1}+A_{2}$, where the operators $A_{0}$, $A_{1}, A_{2}$ are defined by relations (4.1), (4.2). Then the following four assertions are equivalent.

(1) There exists a function $z_{0} \in L^{\infty}\left(\Phi ; \mathbb{R}_{+}\right)$such that $z_{0} \gg A\left(z_{0}\right)$.

(2) The spectral radius of the operator $A$ is less than one.

(3) Problem (1.1), (1.2) is uniquely solvable for arbitrary $q \in L^{\infty}(\Phi ; \mathbb{R})$ and $\alpha \in$ $A C([a, b] ; \mathbb{R}), \beta \in A C([c, d] ; \mathbb{R})$ such that $\alpha^{\prime} \in L^{\infty}([a, b] ; \mathbb{R}), \beta^{\prime} \in L^{\infty}([c, d] ; \mathbb{R})$, and $\alpha(a)=\beta(c)$.

If, in addition, the initial functions $\alpha, \beta$ and the forcing term $q$ are such that

$$
\alpha(a) \geq 0, \quad \alpha^{\prime}(t) \geq 0, \quad \beta^{\prime}(x) \geq 0, \quad q(t, x) \geq 0 \quad \text { for a.e. }(t, x) \in \Phi,
$$

then the solution $u$ to problem (1.1), (1.2) satisfies

$$
\begin{gathered}
u(t, x) \geq 0 \quad \text { for }(t, x) \in \Phi, \\
u^{(1,0)}(t, x) \geq 0 \text { for a.e. } t \in[a, b] \text { and all } x \in[c, d], \\
u^{(0,1)}(t, x) \geq 0 \text { for } t \in[a, b] \text { and a.e. } x \in[c, d] .
\end{gathered}
$$

(4) There exists a function $\gamma \in C^{*}(\Phi ; \mathbb{R})$ such that

$$
\begin{gathered}
r^{(1,1)} \gg e_{0}(r)+e_{1}\left(r^{(1,0)}\right)+e_{2}\left(r^{(0,1)}\right), \\
r(a, c) \geq 0, \\
r^{(1,0)}(t, c) \geq 0 \quad \text { for a.e. } t \in[a, b], \quad r^{(0,1)}(a, x) \geq 0 \quad \text { for a.e. } x \in[c, d], \\
r^{(1,1)}(t, x) \geq 0 \quad \text { for a.e. }(t, x) \in \Phi .
\end{gathered}
$$



following.

For Volterra-type operators $\ell_{0}, \ell_{1}$, and $\ell_{2}$, we derive from the previous theorem the

Corollary 4.5. Let $\ell_{0}, \ell_{1}$, and $\ell_{2}$ be positive $(a, c)$-Volterra, $c$-Volterra, and a-Volterra operators, respectively, such that the inequalities

$$
\ell_{1}(y)(t, x) \leq y(t) \ell_{1}(1)(t, x) \text { for a.e. }(t, x) \in \boldsymbol{\Phi}
$$

(here, $\ell_{1}(y)$ means $\ell_{1}(\bar{y})$ in which $\bar{y}(t, x)=y(t)$ for a.e. $t \in[a, b]$ and all $x \in[c, d]$ ) and

$$
\ell_{2}(z)(t, x) \leq z(x) \ell_{2}(1)(t, x) \quad \text { for a.e. }(t, x) \in \boldsymbol{D}
$$

(by $\ell_{2}(z)$ we mean $\ell_{2}(\bar{z})$, where $\bar{z}(t, x)=z(x)$ for all $t \in[a, b]$ and a.e. $x \in[c, d]$ ) hold for every $y \in L^{\infty}([a, b] ; \mathbb{R})$ and $z \in L^{\infty}([c, d] ; \mathbb{R})$.

Then problem (1.1), (1.2) is uniquely solvable for arbitrary $q \in L^{\infty}(\Phi ; \mathbb{R})$ and $\alpha \in$ $A C([a, b] ; \mathbb{R}), \beta \in A C([c, d] ; \mathbb{R})$ such that $\alpha^{\prime} \in L^{\infty}([a, b] ; \mathbb{R}), \beta^{\prime} \in L^{\infty}([c, d] ; \mathbb{R})$, and $\alpha(a)=\beta(c)$. If, in addition, the initial functions $\alpha, \beta$ and the forcing term $q$ are such that relations (4.7) hold, then the solution $u$ to problem (1.1), (1.2) satisfies inequalities (4.8).

Following our previous results concerning the case, where $\ell_{1}=0$ and $\ell_{2}=0$ (see [18]), we can introduce the following.

Definition 4.6. Let $\ell_{0}: C(\boldsymbol{\Phi} ; \mathbb{R}) \rightarrow L^{\infty}(\boldsymbol{\Phi} ; \mathbb{R}), \ell_{1}: Z_{[2]}(\boldsymbol{\Phi} ; \mathbb{R}) \rightarrow L^{\infty}(\boldsymbol{\Phi} ; \mathbb{R})$, and $\ell_{2}:$ $Z_{[1]}(\Phi ; \mathbb{R}) \rightarrow L^{\infty}(\Phi ; \mathbb{R})$. We say that the triplet $\left(\ell_{0}, \ell_{1}, \ell_{2}\right)$ belongs to the set $S_{a c}^{\prime}$ if the implication

$$
\begin{aligned}
& u \in C^{*}(\boldsymbol{\Phi} ; \mathbb{R}), \\
& u^{(1,1)}(t, x) \geq \ell_{0}(u)(t, x)+\ell_{1}\left(u^{(1,0)}\right)(t, x) \\
&+\ell_{2}\left(u^{(0,1)}\right)(t, x), \text { for a.e. }(t, x) \in \Phi, \\
& u(a, c) \geq 0, \\
& u^{(1,0)}(t, c) \geq 0 \text { for a.e. } t \in[a, b] \\
& u^{(0,1)}(a, x) \geq 0 \text { for a.e. } x \in[c, d] \\
& \Longrightarrow u \text { satisfies }(4.8)
\end{aligned}
$$

holds.

Remark 4.7. If $\left(\ell_{0}, \ell_{1}, \ell_{2}\right) \in \mathcal{S}_{a c}^{\prime}$, we usually say that a certain theorem on differential inequalities holds for (1.1). It should be noted here that there is another terminology which says that a certain maximum principle holds for (1.1) if the inclusion $\left(\ell_{0}, \ell_{1}, \ell_{2}\right) \in \mathcal{S}_{a c}^{\prime}$ is fulfilled.

Theorem 4.4 immediately yields the following.

Corollary 4.8. If one of assertions (1)-(4) stated in Theorem 4.4 holds then $\left(\ell_{0}, \ell_{1}, \ell_{2}\right) \in \mathcal{S}_{a c}^{\prime}$. 
Remark 4.9. The inclusion $\left(\ell_{0}, \ell_{1}, \ell_{2}\right) \in S_{a c}^{\prime}$ ensures that every solution $u$ to problem (1.1), (1.2) with (4.7) satisfies relations (4.8). However, we do not know whether this inclusion also guarantees the unique solvability of problem (1.1), (1.2) for arbitrary $q, \alpha$, and $\beta$. Consequently, we cannot reverse the assertion of the previous corollary.

The reason lays in the question whether the Fredholm alternative holds for problem (1.1), (1.2) or not. In fact, we are not able to prove compactness of the operator $A$ appearing in Theorem 4.4 which plays a crucial role in the proofs of the Fredholm alternative for problem (1.1), (1.2) as well as a continuous dependence of its solutions on the initial data and parameters.

Now we apply general results to (3.2) with argument deviations in which coefficients $p_{0}, p_{1}, p_{2}, q \in L^{\infty}(\boldsymbol{\Phi} ; \mathbb{R})$ and argument deviations $\tau_{0}, \tau_{2}: \Phi \rightarrow[a, b], \mu_{0}, \mu_{1}: \Phi \rightarrow[c, d]$ are measurable functions.

As a consequence of Corollary 4.2 we obtain the following.

Corollary 4.10. If the inequality

$$
(b-a)(d-c)\left\|p_{0}\right\|_{L^{\infty}}+(d-c)\left\|p_{1}\right\|_{L^{\infty}}+(b-a)\left\|p_{2}\right\|_{L^{\infty}}<1
$$

holds, then problem (3.2), (1.2) is uniquely solvable for arbitrary $q \in L^{\infty}(\boldsymbol{\Phi} ; \mathbb{R})$ and $\alpha \in$ $A C([a, b] ; \mathbb{R}), \beta \in A C([c, d] ; \mathbb{R})$ such that $\alpha^{\prime} \in L^{\infty}([a, b] ; \mathbb{R}), \beta^{\prime} \in L^{\infty}([c, d] ; \mathbb{R})$, and $\alpha(a)=\beta(c)$.

If the coefficients $p_{0}, p_{1}, p_{2}$ in the previous corollary are non-negative then the assertion of the corollary follows also from implication $(4) \Rightarrow(3)$ of Theorem 4.4. More precisely, the following statement holds.

Corollary 4.11. Let $p_{0}, p_{1}, p_{2} \in L^{\infty}\left(\Phi ; \mathbb{R}_{+}\right)$and

$$
\begin{gathered}
\text { ess } \sup \left\{p_{0}(t, x)\left(\tau_{0}(t, x)-a\right)\left(\mu_{0}(t, x)-c\right)+p_{1}(t, x)\left(\mu_{1}(t, x)-c\right)\right. \\
\left.+p_{2}(t, x)\left(\tau_{2}(t, x)-a\right):(t, x) \in \Phi\right\}<1
\end{gathered}
$$

Then problem (3.2), (1.2) is uniquely solvable for arbitrary $q \in L^{\infty}(\boldsymbol{\Phi} ; \mathbb{R})$ and $\alpha \in A C([a, b] ; \mathbb{R})$, $\beta \in A C([c, d] ; \mathbb{R})$ such that $\alpha^{\prime} \in L^{\infty}([a, b] ; \mathbb{R}), \beta^{\prime} \in L^{\infty}([c, d] ; \mathbb{R})$, and $\alpha(a)=\beta(c)$. If, in addition, the initial functions $\alpha, \beta$, and the forcing term $q$ are such that relations (4.7) hold, then the solution $u$ to problem (3.2), (1.2) satisfies inequalities (4.8).

Finally, Corollary 4.5 implies the following.

Corollary 4.12. Let $p_{0}, p_{1}, p_{2} \in L^{\infty}\left(\Phi ; \mathbb{R}_{+}\right)$and argument deviations $\tau_{0}, \mu_{0}, \mu_{1}$, and $\tau_{2}$ satisfy inequalities (2.26), (2.32), and (2.33). Then problem (3.2), (1.2) is uniquely solvable for arbitrary $q \in L^{\infty}(\Phi ; \mathbb{R})$ and $\alpha \in A C([a, b] ; \mathbb{R}), \beta \in A C([c, d] ; \mathbb{R})$ such that $\alpha^{\prime} \in L^{\infty}([a, b] ; \mathbb{R}), \beta^{\prime} \in$ $L^{\infty}([c, d] ; \mathbb{R})$, and $\alpha(a)=\beta(c)$. If, in addition, the initial functions $\alpha, \beta$ and the forcing term $q$ are such that relations (4.7) hold, then the solution $u$ to problem (3.2), (1.2) satisfies inequalities (4.8).

The assumptions of the previous corollary require, in fact, that (3.2) is delayed in all its deviating arguments. Observe that in the case, where (3.3) holds, the inequalities (2.26), (2.32), and (2.33) are satisfied trivially and Corollary 4.12 thus conform to the results well 
known for (3.1). The following statements show that the assertion of Corollary 4.12 remains true if the deviations $\tau_{0}, \mu_{0}, \mu_{1}$, and $\tau_{2}$ are not necessarily delays but the differences

$$
\tau_{k}(t, x)-t, \quad \mu_{j}(t, x)-x \quad(k=0,2, j=0,1)
$$

are small enough, that is, if (3.2) with deviating arguments is "close" to (3.1).

Corollary 4.13. Let $p_{0}, p_{1}, p_{2} \in L^{\infty}\left(\boldsymbol{\Phi} ; \mathbb{R}_{+}\right), p_{k} \neq \equiv 0(k=1,2)$, and

$$
\begin{aligned}
& \text { ess sup }\left\{\int_{t}^{\tau_{0}(t, x)} \int_{c}^{\mu_{0}(t, x)} p_{0}(s, \eta) \mathrm{d} \eta \mathrm{d} s+\int_{a}^{t} \int_{x}^{\mu_{0}(t, x)} p_{0}(s, \eta) \mathrm{d} \eta \mathrm{d} s\right. \\
& \left.+2\left\|p_{2}\right\|_{L^{\infty}}\left(\tau_{0}(t, x)-t\right)+2\left\|p_{1}\right\|_{L^{\infty}}\left(\mu_{0}(t, x)-x\right):(t, x) \in \Phi\right\} \leq \frac{\omega}{\mathrm{e}}\left(1+\ln \frac{1}{\omega}\right), \\
& \text { ess } \sup \left\{\int_{a}^{t} \int_{x}^{\mu_{1}(t, x)} p_{0}(s, \eta) \mathrm{d} \eta \mathrm{d} s+2\left\|p_{1}\right\|_{L^{\infty}}\left(\mu_{1}(t, x)-x\right):(t, x) \in \Phi\right\}<\frac{\omega}{\mathrm{e}} \\
& \text { ess } \sup \left\{\int_{t}^{\tau_{2}(t, x)} \int_{c}^{x} p_{0}(s, \eta) \mathrm{d} \eta \mathrm{d} s+2\left\|p_{2}\right\|_{L^{\infty}}\left(\tau_{2}(t, x)-t\right):(t, x) \in \Phi\right\}<\frac{\omega}{\mathrm{e}},
\end{aligned}
$$

where

$$
\omega=\frac{2 \min \left\{\left\|p_{1}\right\|_{L^{\infty}}\left\|p_{2}\right\|_{L^{\infty}}\right\}}{\left\|p_{0}\right\|_{L^{\infty}} \max \{b-a, d-c\}+2 \min \left\{\left\|p_{1}\right\|_{L^{\infty}}\left\|p_{2}\right\|_{L^{\infty}}\right\}} .
$$

Then the assertion of Corollary 4.12 holds.

\section{Auxiliary Statements and Proofs of Main Results}

The proofs use several auxiliary statements given in the next subsection.

\subsection{Auxiliary Statements}

Remember that, for given operators $\ell_{0}, \ell_{1}$, and $\ell_{2}$, the operators $A_{0}, A_{1}$, and $A_{2}$ are defined by relations (4.1), (4.2). Moreover, having $q \in L^{\infty}(\boldsymbol{\Phi} ; \mathbb{R})$ and $\alpha \in A C([a, b] ; \mathbb{R}), \beta \in A C([c, d] ; \mathbb{R})$ such that $\alpha^{\prime} \in L^{\infty}([a, b] ; \mathbb{R}), \beta^{\prime} \in L^{\infty}([c, d] ; \mathbb{R})$, and $\alpha(a)=\beta(c)$, we put

$$
y=\ell_{0}(-\alpha(a)+\alpha+\beta)+\ell_{1}\left(\alpha^{\prime}\right)+\ell_{2}\left(\beta^{\prime}\right)+q
$$

(by $\ell_{0}\left(-\alpha(a)+\alpha+\beta\right.$ ) the authors understand $\ell_{0}(\sigma)$ in which $\sigma(t, x)=-\alpha(a)+\alpha(t)+\beta(x)$ for $(t, x) \in \Phi$. Similarly, $\ell_{1}\left(\alpha^{\prime}\right)$ (resp., $\left.\ell_{2}\left(\beta^{\prime}\right)\right)$ means $\ell_{1}\left(\alpha_{0}\right)$ (resp., $\ell_{2}\left(\beta_{0}\right)$ ), where $\alpha_{0}(t, x)=\alpha^{\prime}(t)$ for a.e. $t \in[a, b]$ and all $x \in[c, d]$ (resp., $\beta_{0}(t, x)=\beta^{\prime}(x)$ for all $t \in[a, b]$ and a.e. $\left.x \in[c, d]\right)$ ). Clearly, $y \in L^{\infty}(\Phi ; \mathbb{R})$. 
Lemma 5.1. If $u$ is a solution to problem (1.1), (1.2) then $u^{(1,1)}$ is a solution to the equation

$$
z=\left(A_{0}+A_{1}+A_{2}\right)(z)+y
$$

in the space $L^{\infty}(\Phi ; \mathbb{R})$, where the operators $A_{0}, A_{1}$, and $A_{2}$ are defined by relations (4.1), (4.2) and the function $y$ is given by formula (5.1).

Conversely, if $z$ is a solution to $(5.2)$ in the space $L^{\infty}(\boldsymbol{\Phi} ; \mathbb{R})$ with the operators $A_{0}, A_{1}$, and $A_{2}$ defined by relations (4.1), (4.2) and the function y given by formula (5.1), then

$$
u(t, x)=-\alpha(a)+\alpha(t)+\beta(x)+\int_{a}^{t} \int_{c}^{x} z(s, \eta) \mathrm{d} \eta \mathrm{d} s \quad \text { for }(t, x) \in \Phi
$$

is a solution to problem (1.1), (1.2).

Proof. If $u$ is a solution to problem (1.1), (1.2) then, by virtue of Remark 2.4, we get $u^{(1,1)} \in$ $L^{\infty}(\Phi ; \mathbb{R})$,

$$
\begin{array}{r}
u(t, x)=-\alpha(a)+\alpha(t)+\beta(x)+\int_{a}^{t} \int_{c}^{x} u^{(1,1)}(s, \eta) \mathrm{d} \eta \mathrm{d} s \quad \text { for }(t, x) \in \Phi, \\
u^{(1,0)}(t, x)=\alpha^{\prime}(t)+\int_{c}^{x} u^{(1,1)}(t, \eta) \mathrm{d} \eta \quad \text { for a.e. } t \in[a, b] \text { and all } x \in[c, d], \\
u^{(0,1)}(t, x)=\beta^{\prime}(x)+\int_{a}^{t} u^{(1,1)}(s, x) \mathrm{d} s \quad \text { for all } t \in[a, b] \text { and a.e. } x \in[c, d] .
\end{array}
$$

Consequently, (1.1) yields that

$$
u^{(1,1)}=\left(A_{0}+A_{1}+A_{2}\right)\left(u^{(1,1)}\right)+y
$$

where the operators $A_{0}, A_{1}$, and $A_{2}$ are defined by relations (4.1), (4.2) and the function $y$ is given by formula (5.1).

Conversely, let $z$ be a solution to $(5.2)$ in the space $L^{\infty}(\Phi ; \mathbb{R})$ with the operators $A_{0}, A_{1}$, and $A_{2}$ defined by relations (4.1), (4.2) and the function $y$ given by formula (5.1). Moreover, let the function $u$ be defined by relation (5.3), that is,

$$
u(t, x)=\alpha(a)+\int_{a}^{t} \alpha^{\prime}(s) \mathrm{d} s+\int_{c}^{x} \beta^{\prime}(\eta) \mathrm{d} \eta+\int_{a}^{t} \int_{c}^{x} z(s, \eta) \mathrm{d} \eta \mathrm{d} s \quad \text { for }(t, x) \in \Phi .
$$


Then the function $u$ belongs to the set $C^{*}(\boldsymbol{\Phi} ; \mathbb{R})$ and verifies initial conditions (1.2). Furthermore, by using Lemma 2.3, we get

$$
\begin{aligned}
& u^{(1,0)}(t, x)=\alpha^{\prime}(t)+\int_{c}^{x} z(t, \eta) \mathrm{d} \eta \text { for a.e. } t \in[a, b] \text { and all } x \in[c, d], \\
& u^{(0,1)}(t, x)=\beta^{\prime}(x)+\int_{a}^{t} z(s, x) \mathrm{d} s \text { for all } t \in[a, b] \text { and a.e. } x \in[c, d], \\
& u^{(1,1)}(t, x)=z(t, x) \quad \text { for a.e. }(t, x) \in \boldsymbol{\Phi} .
\end{aligned}
$$

Consequently, (5.2) implies that $u$ is also a solution to (1.1).

Now we recall some definitions from the theory of linear operators leaving invariant a cone in a Banach space (see, e.g., $[24,25]$ and references therein).

Definition 5.2. A nonempty closed set $K$ in a Banach space $X$ is called a cone if the following conditions are satisfied:

(i) $x+y \in K$ for all $x, y \in K$,

(ii) $\lambda x \in K$ for all $x \in K$ and an arbitrary $\lambda \geq 0$,

(iii) if $x \in K$ and $-x \in K$ then $x=0$.

Remark 5.3. In the original terminology introduced by KreInn and Rutman [25], a set $K$ satisfying conditions (i) and (ii) of Definition 5.2 is called a linear semigroup.

Definition 5.4. We say that a cone $K \subseteq X$ is solid if its interior $\operatorname{Int} K$ is nonempty.

Remark 5.5. The presence of a cone $K$ in a Banach space $X$ allows one to introduce a natural partial ordering there. More precisely, two elements $x_{1}, x_{2} \in X$ are said to be in the relation $x_{2} \geq_{K} x_{1}$ if and only if they satisfy the inclusion $x_{2}-x_{1} \in K$. If, moreover, $K$ is a solid cone then we write $x_{2} \gg_{K} x_{1}$ if and only if $x_{2}-x_{1} \in$ Int $K$.

Definition 5.6. A cone $K \subseteq X$ is said to be normal if there is a constant $N \geq 0$ such that, for every $x, y \in X$ with the property $0 \leq_{K} x \leq_{K} y$, the relation $\|x\|_{X} \leq N\|y\|_{X}$ holds.

The proof of the main part of Theorem 4.4 is based on the following result.

Lemma 5.7 (see [24, Theorem 5.6]). Let $K$ be a normal and solid cone in a Banach space $X$ and the operator $A: X \rightarrow X$ leave invariant the cone $K$, that is, $A(K) \subseteq K$. If there exists a constant $\delta>0$ and an element $x_{0} \in \operatorname{Int} K$ such that $\delta x_{0}-A\left(x_{0}\right) \in \operatorname{Int} K$, then the spectral radius of the operator $A$ is less than $\delta$.

Finally, we establish three lemmas dealing with Volterra type operators which we need to prove Corollary 4.5 .

Lemma 5.8. Let $\ell_{0}: C(\Phi ; \mathbb{R}) \rightarrow L^{\infty}(\Phi ; \mathbb{R})$ be a positive $(a, c)$-Volterra operator. Then, for any function $\gamma \in C(\Phi ; \mathbb{R})$ satisfying

$$
\gamma\left(t_{1}, x_{1}\right) \leq \gamma\left(t_{2}, x_{2}\right) \text { for } a \leq t_{1} \leq t_{2} \leq b, c \leq x_{1} \leq x_{2} \leq d \text {, }
$$


one has

$$
\ell_{0}(\gamma)(t, x) \leq \ell_{0}(1)(t, x) \gamma(t, x) \quad \text { for a.e. }(t, x) \in \Phi \text {. }
$$

Proof. We first show that, for any $(t, x) \in] a, b] \times] c, d]$, we have

$$
\ell_{0}(\gamma)(s, \eta) \leq \ell_{0}(1)(s, \eta) \gamma(t, x) \quad \text { for a.e. }(s, \eta) \in[a, t] \times[c, x]
$$

Indeed, let $(t, x) \in] a, b] \times] c, d]$ be arbitrary but fixed. Put

$$
\gamma_{0}(s, \eta)=\gamma(\min \{s, t\}, \min \{\eta, x\}) \text { for }(s, \eta) \in \Phi \text {. }
$$

Then, clearly $\gamma_{0} \in C(\Phi ; \mathbb{R})$,

$$
\begin{gathered}
\gamma_{0}(s, \eta) \leq \gamma(t, x) \text { for }(s, \eta) \in \Phi, \\
\gamma_{0}(s, \eta)=\gamma(s, \eta) \text { for }(s, \eta) \in[a, t] \times[c, x] .
\end{gathered}
$$

Since the operator $\ell_{0}$ is positive, we obtain

$$
\ell_{0}\left(\gamma_{0}\right)(s, \eta) \leq \ell_{0}(\gamma(t, x))(s, \eta)=\gamma(t, x) \ell_{0}(1)(s, \eta) \quad \text { for a.e. }(s, \eta) \in \Phi \text {. }
$$

On the other hand, the operator $\ell_{0}$ is supposed to be an $(a, c)$-Volterra one which guarantees the equality

$$
\ell_{0}\left(\gamma_{0}\right)(s, \eta)=\ell_{0}(\gamma)(s, \eta) \text { for a.e. }(s, \eta) \in[a, t] \times[c, x]
$$

and thus the desired relation (5.10) holds.

Now we put

$$
\begin{gathered}
u(t, x)=\int_{a}^{t} \int_{c}^{x} \ell_{0}(\gamma)(s, \eta) \mathrm{d} \eta \mathrm{d} s, \\
v(t, x)=\int_{a}^{t} \int_{c}^{x} \ell_{0}(1)(s, \eta) \mathrm{d} \eta \mathrm{d} s \quad \text { for }(t, x) \in \Phi .
\end{gathered}
$$

It follows from Lemma 2.3 that there exists a set $\left.\left.E_{1} \subseteq\right] a, b\right]$, meas $E_{1}=b-a$, such that

$$
\begin{aligned}
& u^{(1,0)}(t, x)=\int_{c}^{x} \ell_{0}(\gamma)(t, \eta) \mathrm{d} \eta \quad \text { for } t \in E_{1}, x \in[c, d], \\
& v^{(1,0)}(t, x)=\int_{c}^{x} \ell_{0}(1)(t, \eta) \mathrm{d} \eta \quad \text { for } t \in E_{1}, x \in[c, d],
\end{aligned}
$$


and, moreover, there is a set $\left.\left.E \subseteq E_{1} \times\right] c, d\right]$, meas $E=(b-a)(d-c)$, with the properties

$$
u^{(1,1)}(t, x)=\ell_{0}(\gamma)(t, x), \quad v^{(1,1)}(t, x)=\ell_{0}(1)(t, x) \quad \text { for }(t, x) \in E .
$$

Let $(t, x) \in E$ be arbitrary but fixed. Then, relation (5.10) yields

$$
\frac{1}{h k} \int_{t-h}^{t} \int_{x-k}^{x} \ell_{0}(\gamma)(s, \eta) \mathrm{d} \eta \mathrm{d} s \leq \frac{\gamma(t, x)}{h k} \int_{t-h}^{t} \int_{x-k}^{x} \ell_{0}(1)(s, \eta) \mathrm{d} \eta \mathrm{d} s
$$

for $h \in] 0, t-a]$ and $k \in] 0, x-c]$, whence we get

$$
\begin{array}{r}
\frac{1}{k}\left[\frac{u(t, x)-u(t-h, x)}{h}-\frac{u(t, x-k)-u(t-h, x-k)}{h}\right] \\
\leq \frac{r(t, x)}{k}\left[\frac{v(t, x)-v(t-h, x)}{h}-\frac{v(t, x-k)-v(t-h, x-k)}{h}\right] \\
\text { for } h \in] 0, t-a], k \in] 0, x-c]
\end{array}
$$

For any $k \in] 0, x-c]$ fixed, we pass to the limit $h \rightarrow 0+$ in the latter inequality and thus, in view of equalities (5.16), we get

$$
\frac{1}{k}\left[u^{(1,0)}(t, x)-u^{(1,0)}(t, x-k)\right] \leq \frac{r(t, x)}{k}\left[v^{(1,0)}(t, x)-v^{(1,0)}(t, x-k)\right]
$$

for $k \in] 0, x-c]$. Now, letting $k \rightarrow 0+$ in the previous relation and using equalities (5.17) give

$$
\ell_{0}(\gamma)(t, x)=u^{(1,1)}(t, x) \leq \gamma(t, x) v^{(1,1)}(t, x)=\gamma(t, x) \ell_{0}(1)(t, x) .
$$

That is, the desired inequality (5.9) holds because $(t, x) \in E$ was arbitrary.

Lemma 5.9. Let $\ell_{2}: Z_{[1]}(\Phi ; \mathbb{R}) \rightarrow L^{\infty}(\Phi ; \mathbb{R})$ be a positive a-Volterra operator such that inequality (4.14) holds for every $z \in L^{\infty}([c, d] ; \mathbb{R})$. Then, for any function $\gamma \in Z_{[1]}(\boldsymbol{\Phi} ; \mathbb{R})$ with the property

$$
\gamma\left(t_{1}, x\right) \leq \gamma\left(t_{2}, x\right) \text { for } a \leq t_{1} \leq t_{2} \leq b \text { and a.e. } x \in[c, d] \text {, }
$$

one has

$$
\ell_{2}(\gamma)(t, x) \leq \ell_{2}(1)(t, x) \gamma(t, x) \text { for a.e. }(t, x) \in \Phi \text {. }
$$

Proof. Let $E_{1} \subseteq[c, d]$, meas $E_{1}=d-c$, be a set such that, for any $x \in E_{1}$, we have $\gamma(\cdot, x) \in$ $C([a, b] ; \mathbb{R})$ and

$$
\gamma\left(t_{1}, x\right) \leq \gamma\left(t_{2}, x\right) \text { for } a \leq t_{1} \leq t_{2} \leq b .
$$


We first show that the relation

$$
\ell_{2}(\gamma)(s, x) \leq \ell_{2}(1)(s, x) \gamma(t, x) \text { for a.e. }(s, x) \in[a, t] \times[c, d]
$$

holds for every $t \in] a, b]$. Indeed, let $t \in] a, b]$ be arbitrary but fixed. Put

$$
\begin{gathered}
\gamma_{0}(s, x)=\gamma(\min \{s, t\}, x) \text { for } s \in[a, b], x \in E_{1}, \\
\gamma_{1}(x)=\gamma(t, x) \text { for } x \in E_{1} .
\end{gathered}
$$

Then, clearly, $\gamma_{0} \in Z_{[1]}(\Phi ; \mathbb{R}), \gamma_{1} \in L^{\infty}([c, d] ; \mathbb{R})$,

$$
\begin{aligned}
& \gamma_{0}(s, x) \leq \gamma_{1}(x) \text { for } s \in[a, b], x \in E_{1}, \\
& \gamma_{0}(s, x)=\gamma(s, x) \text { for } s \in[a, t], x \in E_{1} .
\end{aligned}
$$

Since the operator $\ell_{2}$ is positive and satisfies condition (4.14), we obtain

$$
\ell_{2}\left(\gamma_{0}\right)(s, x) \leq \ell_{2}\left(\gamma_{1}\right)(s, x) \leq \gamma_{1}(x) \ell_{2}(1)(s, x) \quad \text { for a.e. }(s, x) \in \boldsymbol{D}
$$

(by $\ell_{2}\left(\gamma_{1}\right)$ the authors mean $\ell_{2}\left(\bar{\gamma}_{1}\right)$, where $\bar{\gamma}_{1}(s, x)=\gamma_{1}(x)$ for all $s \in[a, b]$ and $\left.x \in E_{1}\right)$. On the other hand, the operator $\ell_{2}$ is supposed to be an $a$-Volterra one which guarantees the equality

$$
\ell_{2}\left(\gamma_{0}\right)(s, x)=\ell_{2}(\gamma)(s, x) \text { for a.e. }(s, x) \in[a, t] \times[c, d]
$$

and thus desired relation (5.25) holds for every $t \in] a, b]$. It means that, for any $t \in] a, b]$, there exists a set $A_{t} \subseteq[c, d]$ with meas $A_{t}=d-c$ such that

$$
\ell_{2}(\gamma)(s, x) \leq \ell_{2}(1)(s, x) \gamma(t, x) \text { for } x \in A_{t}, s \in B_{t}(x) \text {, }
$$

where, for each $x \in A_{t}$, we have $B_{t}(x) \subseteq[a, b]$ with meas $B_{t}(x)=t-a$.

Put $E_{2}=\cap_{t \in C} A_{t}$, where $\left.\left.C=\right] a, b\right] \cap \mathbb{Q}$. Clearly, meas $E_{2}=d-c$ because the set $C$ is countable. Moreover, relation (5.30) yields that

$$
\int_{t_{0}}^{t} \ell_{2}(\gamma)(s, x) \mathrm{d} s \leq \gamma(t, x) \int_{t_{0}}^{t} \ell_{2}(1)(s, x) \mathrm{d} s \quad \text { for } x \in E_{2}, t \in C, t_{0} \in[a, t] .
$$

Let $t \in] a, b], t_{0} \in\left[a, t\left[\right.\right.$, and $x \in E_{1} \cap E_{2}$ be arbitrary but fixed. Then there exists a sequence $\left\{t_{n}\right\}_{n=1}^{+\infty} \subset\left[t_{0}, b\right] \cap \mathbb{Q}$ such that $t_{n} \rightarrow t$ as $n \rightarrow+\infty$. It follows from relation (5.31) that

$$
\int_{t_{0}}^{t_{n}} \ell_{2}(\gamma)(s, x) \mathrm{d} s \leq \gamma\left(t_{n}, x\right) \int_{t_{0}}^{t_{n}} \ell_{2}(1)(s, x) \mathrm{d} s \quad \text { for } n \in \mathbb{N},
$$


whence we get

$$
\int_{t_{0}}^{t} \ell_{2}(\gamma)(s, x) \mathrm{d} s \leq \gamma(t, x) \int_{t_{0}}^{t} \ell_{2}(1)(s, x) \mathrm{d} s
$$

Consequently, we have proved that

$$
\int_{t_{0}}^{t} \ell_{2}(\gamma)(s, x) \mathrm{d} s \leq \gamma(t, x) \int_{t_{0}}^{t} \ell_{2}(1)(s, x) \mathrm{d} s, \quad \text { for } x \in E_{1} \cap E_{2}, a \leq t_{0}<t \leq b .
$$

Now we put

$$
u(t, x)=\int_{a}^{t} \ell_{2}(\gamma)(s, x) \mathrm{d} s, \quad v(t, x)=\int_{a}^{t} \ell_{2}(1)(s, x) \mathrm{d} s \quad \text { for } t \in[a, b], x \in E_{1} \cap E_{2} .
$$

Lemma 2.3 guarantees that there exists a set $E \subseteq] a, b] \times\left(E_{1} \cap E_{2}\right)$ such that meas $E=(b-$ a) $(d-c)$ and

$$
u^{(1,0)}(t, x)=\ell_{2}(\gamma)(t, x), \quad v^{(1,0)}(t, x)=\ell_{2}(1)(t, x) \quad \text { for }(t, x) \in E .
$$

Let $(t, x) \in E$ be arbitrary but fixed. We choose a sequence $\left\{h_{n}\right\}_{n=1}^{+\infty}$ of numbers from the interval $] 0, t-a$ ] such that

$$
\lim _{n \rightarrow+\infty} h_{n}=0
$$

Then relation (5.34) yields that

$$
\frac{1}{h_{n}} \int_{t-h_{n}}^{t} \ell_{2}(\gamma)(s, x) \mathrm{d} s \leq \frac{\gamma(t, x)}{h_{n}} \int_{t-h_{n}}^{t} \ell_{2}(1)(s, x) \mathrm{d} s \quad \text { for } n \in \mathbb{N} .
$$

Letting $n \rightarrow+\infty$ in the previous relation and using equalities (5.36) give

$$
\ell_{2}(\gamma)(t, x)=u^{(1,0)}(t, x) \leq \gamma(t, x) v^{(1,0)}(t, x)=\gamma(t, x) \ell_{2}(1)(t, x) .
$$

Consequently, desired inequality (5.23) holds because $(t, x) \in E$ was arbitrary.

Lemma 5.10. Let $\ell_{1}: Z_{[2]}(\Phi ; \mathbb{R}) \rightarrow L^{\infty}(\Phi ; \mathbb{R})$ be a positive c-Volterra operator such that inequality (4.13) holds for every $y \in L^{\infty}([a, b] ; \mathbb{R})$. Then, for any function $\gamma \in Z_{[2]}(\Phi ; \mathbb{R})$ with the property

$$
\gamma\left(t, x_{1}\right) \leq \gamma\left(t, x_{2}\right) \text { for a.e. } t \in[a, b] \text { and all } c \leq x_{1} \leq x_{2} \leq d \text {, }
$$

one has

$$
\ell_{1}(\gamma)(t, x) \leq \ell_{1}(1)(t, x) \gamma(t, x) \quad \text { for a.e. }(t, x) \in \Phi \text {. }
$$


Proof. Lemma can be proven analogously as Lemma 5.9 by exchanging the roles of the variables $t$ and $x$.

\subsection{Proofs of Main Results}

Now we are in a position to prove the main results stated in Section 4.

Proof of Theorem 4.1. Since the spectral radius of the linear bounded operator $A: L^{\infty}(\Phi ; \mathbb{R}) \rightarrow$ $L^{\infty}(\boldsymbol{\Phi} ; \mathbb{R})$ is less than one, (5.2) has a unique solution for an arbitrary $y \in L^{\infty}(\boldsymbol{\Phi} ; \mathbb{R})$ and thus, in view of Lemma 5.1, problem (1.1), (1.2) has a unique solution for every $q \in L^{\infty}(\Phi ; \mathbb{R})$ and $\alpha \in A C([a, b] ; \mathbb{R}), \beta \in A C([c, d] ; \mathbb{R})$ such that $\alpha^{\prime} \in L^{\infty}([a, b] ; \mathbb{R}), \beta^{\prime} \in L^{\infty}([c, d] ; \mathbb{R})$, and $\alpha(a)=\beta(c)$.

Proof of Corollary 4.2. It is easy to show that the norms of the linear bounded operators $A_{0}, A_{1}$, $A_{2}: L^{\infty}(\boldsymbol{\Phi} ; \mathbb{R}) \rightarrow L^{\infty}(\boldsymbol{\Phi} ; \mathbb{R})$ defined by relations (4.1), (4.2) satisfy the estimates

$$
\left\|A_{0}\right\| \leq(b-a)(d-c)\left\|\ell_{0}\right\|, \quad\left\|A_{1}\right\| \leq(d-c)\left\|\ell_{1}\right\|, \quad\left\|A_{2}\right\| \leq(b-a)\left\|\ell_{2}\right\| .
$$

Consequently, the assumption (4.3) guarantees that the spectral radius of the operator $A_{0}+$ $A_{1}+A_{2}$ is less than one. Hence, the assertion of the corollary follows from Theorem 4.1.

Proof of Theorem 4.4. To prove the theorem, it is sufficient to show the following four implications.

$(1) \Rightarrow(2)$ : assume that the assertion (1) of the theorem holds. We put $K=L^{\infty}\left(\boldsymbol{\Phi} ; \mathbb{R}_{+}\right)$. It is not difficult to verify that $K$ forms a normal and solid cone in the Banach space $L^{\infty}(\mathbb{\Phi} ; \mathbb{R})$. Moreover, a function $z \in L^{\infty}(\boldsymbol{\Phi} ; \mathbb{R})$ satisfies the relation $z \gg 0$ if and only if the inclusion $z \in$ Int $K$ holds.

On the other hand, by virtue of (4.1) and (4.2), the operator $A$ leaves the cone $K$ invariant, that is, $A(K) \subseteq K$ because the operators $\ell_{0}, \ell_{1}$, and $\ell_{2}$ are supposed to be positive. Therefore, the assumptions $z_{0} \in K$ and $z_{0} \gg A\left(z_{0}\right)$ yield that $z_{0} \gg 0$ as well.

Applying Lemma 5.7 with $X=L^{\infty}(\Phi ; \mathbb{R})$ and $\delta=1$, we obtain the desired assertion (2) of our theorem.

$(2) \Rightarrow(3)$ : assume that the spectral radius of the operator $A$ is less than one. Then, for an arbitrary $y \in L^{\infty}(\boldsymbol{\Phi} ; \mathbb{R})$, (5.2) has a unique solution $z$ and, moreover, this solution admits the series representation

$$
z=y+A(y)+A^{2}(y)+\cdots
$$

Consequently, in view of Lemma 5.1, problem (1.1), (1.2) has a unique solution $u$ for every $q \in L^{\infty}(\Phi ; \mathbb{R})$ and $\alpha \in A C([a, b] ; \mathbb{R}), \beta \in A C([c, d] ; \mathbb{R})$ such that $\alpha^{\prime} \in L^{\infty}([a, b] ; \mathbb{R}), \beta^{\prime} \in$ $L^{\infty}([c, d] ; \mathbb{R})$, and $\alpha(a)=\beta(c)$.

Assume, in addition, that the initial functions $\alpha, \beta$ and the forcing term $q$ are such that relations (4.7) hold. The above-used Lemma 5.1 guarantees that the solution $u$ to problem (1.1), (1.2) admits the integral representation

$$
u(t, x)=\alpha(a)+\int_{a}^{t} \alpha^{\prime}(s) \mathrm{d} s+\int_{c}^{x} \beta^{\prime}(\eta) \mathrm{d} \eta+\int_{a}^{t} \int_{c}^{x} z(s, \eta) \mathrm{d} \eta \mathrm{d} s \quad \text { for }(t, x) \in \boldsymbol{\Phi}
$$


in which $z$ is given by formula (5.43) with $y$ defined by relation (5.1). Clearly, assumption (4.7) yields $y \geq 0$ and thus the series representation (5.43) ensures that $z \geq 0$ because the operator $A$ leaves invariant the cone $L^{\infty}\left(\Phi ; \mathbb{R}_{+}\right)$. Hence, by virtue of Remark 2.4 , desired property (4.8) of the solution $u$ follows from integral representation (5.44).

$(3) \Rightarrow(4)$ : assume that the assertion (3) of the theorem holds. Then, clearly the problem

$$
\begin{gathered}
\gamma^{(1,1)}(t, x)=\ell_{0}(r)(t, x)+\ell_{1}\left(r^{(1,0)}\right)(t, x)+\ell_{2}\left(r^{(0,1)}\right)(t, x)+1, \\
r(t, c)=0 \quad \text { for } t \in[a, b], \quad \gamma(a, x)=0 \quad \text { for } x \in[c, d]
\end{gathered}
$$

has a unique solution $\gamma$ and the function $\gamma \in C^{*}(\mathscr{D} ; \mathbb{R})$ satisfies the inequalities

$$
\begin{aligned}
r(t, x) \geq 0 & \text { for }(t, x) \in \Phi, \\
r^{(1,0)}(t, x) \geq 0 & \text { for a.e. } t \in[a, b] \text { and all } x \in[c, d], \\
\gamma^{(0,1)}(t, x) \geq 0 & \text { for } t \in[a, b] \text { and a.e. } x \in[c, d] .
\end{aligned}
$$

Hence, conditions (4.9)-(4.11) are fulfilled. Moreover, inequality (4.12) follows from (5.45) because the operators $\ell_{0}, \ell_{1}$, and $\ell_{2}$ are positive and $\gamma$ satisfies relations (5.47).

$(4) \Rightarrow(1)$ : assume that there exists a function $\gamma \in C^{*}(\boldsymbol{\Phi} ; \mathbb{R})$ satisfying inequalities (4.9)-(4.12). Then, by virtue of Remark 2.4 , we get $\gamma^{(1,1)} \in L^{\infty}\left(\boldsymbol{\Phi} ; \mathbb{R}_{+}\right)$,

$$
\begin{gathered}
r(t, x) \geq \int_{a}^{t} \int_{c}^{x} \gamma^{(1,1)}(s, \eta) \mathrm{d} \eta \mathrm{d} s \quad \text { for }(t, x) \in \Phi, \\
\gamma^{(1,0)}(t, x) \geq \int_{c}^{x} \gamma^{(1,1)}(t, \eta) \mathrm{d} \eta \quad \text { for a.e. } t \in[a, b] \text { and all } x \in[c, d], \\
r^{(0,1)}(t, x) \geq \int_{a}^{t} \gamma^{(1,1)}(s, x) \mathrm{d} s, \quad \text { for all } t \in[a, b], \text { a.e. } x \in[c, d] .
\end{gathered}
$$

Consequently, assertion (1) of the theorem holds with $z_{0}=r^{(1,1)}$ because the operators $\ell_{0}, \ell_{1}$, and $\ell_{2}$ are positive.

Proof of Corollary 4.5. We put

$$
\gamma(t, x)=\mathrm{e}^{\int_{a}^{t} \int_{c}^{x}\left(1+\ell_{0}(1)(s, \eta)\right) \mathrm{d} \eta \mathrm{d} s+2 g_{2}(t-a)+2 g_{1}(x-c)} \quad \text { for }(t, x) \in \boldsymbol{\Phi}
$$

where $g_{1}=\left\|\ell_{1}(1)\right\|_{L^{\infty}}$ and $g_{2}=\left\|\ell_{2}(1)\right\|_{L^{\infty}}$. It can be verified that $\gamma \in C^{*}(\Phi ; \mathbb{R})$ and satisfies the inequality

$$
\gamma(t, x) \geq 1 \quad \text { for }(t, x) \in \Phi
$$


In view of Lemma 2.3, from (5.49) we get

$$
\begin{aligned}
& \gamma^{(1,0)}(t, x)=\left(\int_{c}^{x}\left(1+\ell_{0}(1)(t, \eta)\right) \mathrm{d} \eta+2 g_{2}\right) \gamma(t, x), \quad \text { for a.e. } t \in[a, b] \text { and all } x \in[c, d], \\
& \gamma^{(0,1)}(t, x)=\left(\int_{a}^{t}\left(1+\ell_{0}(1)(s, x)\right) \mathrm{d} s+2 g_{1}\right) \gamma(t, x), \quad \text { for } t \in[a, b] \text { and a.e. } x \in[c, d] .
\end{aligned}
$$

Therefore, inequalities (4.10) and (4.11) are fulfilled and

$$
r^{(1,0)}(t, x) \geq 0, \quad r^{(0,1)}(t, x) \geq 0 \quad \text { for a.e. }(t, x) \in \Phi
$$

because the operator $\ell_{0}$ is positive. Moreover, by using Lemma 2.3 and inequalities (5.50) and (5.52), it follows from equalities (5.51) that

$$
\begin{aligned}
\gamma^{(1,1)}(t, x)= & \left(1+\ell_{0}(1)(t, x)\right) \gamma(t, x)+\left(\int_{c}^{x}\left(1+\ell_{0}(1)(t, \eta)\right) \mathrm{d} \eta+2 g_{2}\right) r^{(0,1)}(t, x) \\
= & \left(1+\ell_{0}(1)(t, x)\right) \gamma(t, x) \\
& +\frac{1}{2}\left(\int_{a}^{t}\left(1+\ell_{0}(1)(s, x)\right) \mathrm{d} s+2 g_{1}\right) \gamma^{(1,0)}(t, x) \\
& +\frac{1}{2}\left(\int_{c}^{x}\left(1+\ell_{0}(1)(t, \eta)\right) \mathrm{d} \eta+2 g_{2}\right) r^{(0,1)}(t, x) \\
\geq & 1+\ell_{0}(1)(t, x) \gamma(t, x)+g_{1} \gamma^{(1,0)}(t, x)+g_{2} \gamma^{(0,1)}(t, x) \\
\geq & \ell_{0}(1)(t, x) \gamma(t, x)+\ell_{1}(1)(t, x) \gamma^{(1,0)}(t, x)+\ell_{2}(1)(t, x) \gamma^{(0,1)}(t, x)+1,
\end{aligned}
$$

for a.e. $(t, x) \in \Phi$ and thus inequality (4.12) is satisfied.

On the other hand, equalities (5.49), (5.51) guarantee the validity of the inequalities

$$
\begin{gathered}
\gamma\left(t_{1}, x_{1}\right) \leq \gamma\left(t_{2}, x_{2}\right) \quad \text { for } a \leq t_{1} \leq t_{2} \leq b, c \leq x_{1} \leq x_{2} \leq d, \\
\gamma^{(1,0)}\left(t, x_{1}\right) \leq \gamma^{(1,0)}\left(t, x_{2}\right) \quad \text { for a.e. } t \in[a, b] \text { and all } c \leq x_{1} \leq x_{2} \leq d, \\
\gamma^{(0,1)}\left(t_{1}, x\right) \leq \gamma^{(0,1)}\left(t_{2}, x\right) \text { for } a \leq t_{1} \leq t_{2} \leq b \text { and a.e. } x \in[c, d] .
\end{gathered}
$$

Therefore, by using Lemmas 5.8-5.10, from inequality (5.53) we get

$$
\gamma^{(1,1)}(t, x) \geq \ell_{0}(\gamma)(t, x)+\ell_{1}\left(\gamma^{(1,0)}\right)(t, x)+\ell_{2}\left(\gamma^{(0,1)}\right)(t, x)+1 \quad \text { for a.e. }(t, x) \in \boldsymbol{\Phi}
$$

that is, relation (4.9) holds. 
Consequently, we have found a function $\gamma$ satisfying conditions (4.9)-(4.12) and thus the assertion of the corollary follows from Theorem 4.4.

Proof of Corollary 4.10. It is clear that (3.2) is a particular case of (1.1) in which the operators $\ell_{0}, \ell_{1}$, and $\ell_{2}$ are defined by formulas (2.23), (2.28), and (2.29), respectively (see Examples 2.7 and 2.11). Since we have

$$
\left\|\ell_{k}\right\|=\left\|p_{k}\right\|_{L^{\infty}} \text { for } k=0,1,2
$$

the validity of the corollary follows immediately from Corollary 4.2.

Proof of Corollary 4.11. It is clear that (3.2) is a particular case of (1.1) in which the operators $\ell_{0}, \ell_{1}$, and $\ell_{2}$ are defined by formulas (2.23), (2.28), and (2.29), respectively, and that the operators indicated are positive (see Examples 2.7 and 2.11). Moreover, in view of inequality (4.17), there exists $\varepsilon>0$ such that

$$
\begin{aligned}
1 \geq & p_{0}(t, x)\left(\tau_{0}(t, x)-a\right)\left(\mu_{0}(t, x)-c\right)+p_{1}(t, x)\left(\mu_{1}(t, x)-c\right) \\
& +p_{2}(t, x)\left(\tau_{2}(t, x)-a\right)+\varepsilon \text { for a.e. }(t, x) \in \Phi .
\end{aligned}
$$

Therefore, the function $\gamma$ defined by the relation

$$
\gamma(t, x)=(t-a)(x-c) \quad \text { for }(t, x) \in \Phi
$$

satisfies inequalities (4.9)-(4.12) and thus the assertion of the corollary follows from Theorem 4.4.

Proof of Corollary 4.12. It is clear that (3.2) is a particular case of (1.1) in which the operators $\ell_{0}$, $\ell_{1}$, and $\ell_{2}$ are defined by formulas (2.23), (2.28), and (2.29), respectively, and that conditions (4.13) and (4.14) are fulfilled (see Examples 2.7 and 2.11). Moreover, the operators $\ell_{0}, \ell_{1}$ and $\ell_{2}$ are positive and, in view of Remarks 2.9 and 2.13, the operators indicated are, respectively, $(a, c)$-Volterra, $c$-Volterra, and $a$-Volterra ones.

Consequently, the validity of the corollary follows from Corollary 4.5.

Proof of Corollary 4.13. It is clear that (3.2) is a particular case of (1.1) in which the operators $\ell_{0}, \ell_{1}$, and $\ell_{2}$ are defined by formulas (2.23), (2.28), and (2.29), respectively, and that the operators indicated are positive (see Examples 2.7 and 2.11). 
Let $\tilde{p}_{k}=\left\|p_{k}\right\|_{L^{\infty}}(k=0,1,2)$ and $y=\mathrm{e} / \omega$. According to (4.19) and (4.20), there exist numbers $\varepsilon>0$ and $\delta \in] 0,1[$ such that inequalities

$$
\begin{aligned}
& \int_{t}^{\tau_{0}(t, x)} \int_{c}^{\mu_{0}(t, x)}\left(\varepsilon+p_{0}(s, \eta)\right) \mathrm{d} \eta \mathrm{d} s+\int_{a}^{t} \int_{x}^{\mu_{0}(t, x)}\left(\varepsilon+p_{0}(s, \eta)\right) \mathrm{d} \eta \mathrm{d} s \\
& \quad+2 \tilde{p}_{2}\left(\tau_{0}(t, x)-t\right)+2 \tilde{p}_{1}\left(\mu_{0}(t, x)-x\right) \\
& \leq \frac{1}{y} \ln \left(y+\frac{y \delta}{\left.\mathrm{e}^{y\left[\int_{a}^{b} \int_{c}^{d}\left(\varepsilon+p_{0}(s, \eta)\right) \mathrm{d} \eta \mathrm{d} s+2 \tilde{p}_{2}(b-a)+2 \tilde{p}_{1}(d-c)\right]-\delta}\right),}\right. \\
& \int_{a}^{t} \int_{x}^{\mu_{1}(t, x)}\left(\varepsilon+p_{0}(s, \eta)\right) \mathrm{d} \eta \mathrm{d} s+2 \widetilde{p}_{1}\left(\mu_{1}(t, x)-x\right) \\
& \leq \frac{1}{y} \ln \frac{2 \tilde{p}_{2} y}{\left(\varepsilon+\tilde{p}_{0}\right)(d-c)+2 \widetilde{p}_{2}}, \\
& \int_{t}^{\tau_{2}(t, x)} \int_{c}^{x}\left(\varepsilon+p_{0}(s, \eta)\right) \mathrm{d} \eta \mathrm{d} s+2 \widetilde{p}_{2}\left(\tau_{2}(t, x)-t\right) \\
& \leq \frac{1}{y} \ln \frac{2 \tilde{p}_{1} y}{\left(\varepsilon+\tilde{p}_{0}\right)(b-a)+2 \tilde{p}_{1}},
\end{aligned}
$$

hold for a.e. $(t, x) \in \Phi$. Now we put

$$
\gamma(t, x)=\mathrm{e}^{y z(t, x)}-\delta \quad \text { for }(t, x) \in \Phi,
$$

where

$$
z(t, x)=\int_{a}^{t} \int_{c}^{x}\left(\varepsilon+p_{0}(s, \eta)\right) \mathrm{d} \eta \mathrm{d} s+2 \tilde{p}_{2}(t-a)+2 \tilde{p}_{1}(x-c) \quad \text { for }(t, x) \in \Phi .
$$

It can be verified that $\gamma \in C^{*}(\boldsymbol{\Phi} ; \mathbb{R})$ and satisfies

$$
\gamma(t, x) \geq 1-\delta>0 \quad \text { for }(t, x) \in \Phi
$$

In view of Lemma 2.3, from (5.62) we get

$$
\begin{aligned}
& \gamma^{(1,0)}(t, x)=y\left(\int_{c}^{x}\left(\varepsilon+p_{0}(t, \eta)\right) \mathrm{d} \eta+2 \tilde{p}_{2}\right)(\gamma(t, x)+\delta), \quad \text { for a.e. } t \in[a, b] \text { and all } x \in[c, d], \\
& \gamma^{(0,1)}(t, x)=y\left(\int_{a}^{t}\left(\varepsilon+p_{0}(s, x)\right) \mathrm{d} s+2 \tilde{p}_{1}\right)(\gamma(t, x)+\delta), \quad \text { for } t \in[a, b] \text { and a.e. } x \in[c, d] .
\end{aligned}
$$


Therefore, relations (4.10), (4.11), and (5.52) are fulfilled. Moreover, by using Lemma 2.3 and inequalities (5.52) and (5.62), it follows from equalities (5.63) that

$$
\begin{aligned}
r^{(1,1)}(t, x)= & y\left(\varepsilon+p_{0}(t, x)\right)(\gamma(t, x)+\delta)+y\left(\int_{c}^{x}\left(\varepsilon+p_{0}(t, \eta)\right) \mathrm{d} \eta+2 \tilde{p}_{2}\right) r^{(0,1)}(t, x) \\
= & y \varepsilon(\gamma(t, x)+\delta)+y p_{0}(t, x)(\gamma(t, x)+\delta) \\
& +\frac{y}{2}\left(\int_{a}^{t}\left(\varepsilon+p_{0}(s, x)\right) \mathrm{d} s+2 \tilde{p}_{1}\right) r^{(1,0)}(t, x) \\
& +\frac{y}{2}\left(\int_{c}^{x}\left(\varepsilon+p_{0}(t, \eta)\right) \mathrm{d} \eta+2 \tilde{p}_{2}\right) r^{(0,1)}(t, x) \\
\geq & y \varepsilon+p_{0}(t, x) y(\gamma(t, x)+\delta)+y \tilde{p}_{1} \gamma^{(1,0)}(t, x)+y \tilde{p}_{2} \gamma^{(0,1)}(t, x) \\
\geq & p_{0}(t, x) y(\gamma(t, x)+\delta)+p_{1}(t, x) y r^{(1,0)}(t, x)+p_{2}(t, x) y \gamma^{(0,1)}(t, x)+y \varepsilon
\end{aligned}
$$

for a.e. $(t, x) \in \Phi$ and thus inequality (4.12) is satisfied. Now observe that inequalities (5.59) can be rewritten to the forms

$$
\begin{gathered}
z\left(\tau_{0}(t, x), \mu_{0}(t, x)\right)-z(t, x) \leq \frac{1}{y} \ln \left(y+\frac{y \delta}{\mathrm{e}^{y z\left(\tau_{0}(t, x), \mu_{0}(t, x)\right)}-\delta}\right) \\
z\left(t, \mu_{1}(t, x)\right)-z(t, x) \leq \frac{1}{y} \ln \frac{2 \widetilde{p}_{2} y}{\int_{c}^{\mu_{1}(t, x)}\left(\varepsilon+p_{0}(t, \eta)\right) \mathrm{d} \eta+2 \widetilde{p}_{2}}, \\
z\left(\tau_{2}(t, x), x\right)-z(t, x) \leq \frac{1}{y} \ln \frac{2 \tilde{p}_{1} y}{\int_{a}^{\tau_{2}(t, x)}\left(\varepsilon+p_{0}(s, x)\right) \mathrm{d} s+2 \tilde{p}_{1}},
\end{gathered}
$$

for a.e. $(t, x) \in \Phi$ and thus, by using relations (5.60), (5.63), we get

$$
\begin{gathered}
y(\gamma(t, x)+\delta) \geq \gamma\left(\tau_{0}(t, x), \mu_{0}(t, x)\right) \quad \text { for a.e. }(t, x) \in \Phi, \\
y \gamma^{(1,0)}(t, x) \geq \gamma^{(1,0)}\left(t, \mu_{1}(t, x)\right), \quad y \gamma^{(0,1)}(t, x) \geq \gamma^{(0,1)}\left(\tau_{2}(t, x), x\right)
\end{gathered}
$$

for a.e. $(t, x) \in \Phi$. Consequently, it follows from (5.64) that inequality (4.9) holds.

We have constructed a function $\gamma$ satisfying conditions (4.9)-(4.12) and thus the assertion of the corollary follows from Theorem 4.4 .

\section{Acknowledgment}

For A. Lomtatidze and J. Šremr, the research was supported by the Academy of Sciences of the Czech Republic, Institutional Research Plan no. AV0Z10190503. 


\section{References}

[1] S. Kharibegashvili, "Goursat and Darboux type problems for linear hyperbolic partial differential equations and systems," Memoirs on Differential Equations and Mathematical Physics, vol. 4, pp. 1-127, 1995.

[2] V. Lakshmikantham and S. G. Pandit, "The method of upper, lower solutions and hyperbolic partial differential equations," Journal of Mathematical Analysis and Applications, vol. 105, no. 2, pp. 466-477, 1985.

[3] Z. O. Mel'nik and A. D. Myshkis, "A mixed problem for a two-dimensional hyperbolic system of the first order with discontinuous coefficients," Matematicheskii Sbornik, vol. 68, no. 4, pp. 632-638, 1965.

[4] W. Walter, "Über die Differentialgleichung $u_{x y}=f\left(x, y, u, u_{x}, u_{y}\right)$, Teil I," Mathematische Zeitschrift, vol. 71, pp. 308-324, 1959.

[5] W. Walter, "Über die Differentialgleichung $u_{x y}=f\left(x, y, u, u_{x}, u_{y}\right)$, Teil II," Mathematische Zeitschrift, vol. 71, pp. 436-453, 1959.

[6] W. Walter, "Über die Differentialgleichung $u_{x y}=f\left(x, y, u, u_{x}, u_{y}\right)$, Tiel III," Mathematische Zeitschrift, vol. 73 , pp. 268-279, 1960.

[7] S. Walczak, "Absolutely continuous functions of several variables and their application to differential equations," Bulletin of the Polish Academy of Sciences. Mathematics, vol. 35, no. 11-12, pp. 733-744, 1987.

[8] W. Walter, Differential and Integral Inequalities, Springer, Berlin, Germany, 1970.

[9] O. Vejvoda, "Periodic solutions of a linear and weakly nonlinear wave equation in one dimension," Czechoslovak Mathematical Journal, vol. 14, no. 3, pp. 341-382, 1964.

[10] T. Kiguradze, "Some boundary value problems for systems of linear partial differential equations of hyperbolic type," Memoirs on Differential Equations and Mathematical Physics, vol. 1, pp. 1-144, 1994.

[11] F. G. Tricomi, Lezioni sulle equazioni a derivate parziali, Corso di analisi superiore, Editrice Gheroni, Torino, Italy, 1954.

[12] T. I. Kiguradze and T. Kusano, "On ill-posed initial-boundary value problems for higher-order linear hyperbolic equations with two independent variables," Differential Equations, vol. 39, no. 10, pp. 14541470, 2003.

[13] T. I. Kiguradze and T. Kusano, "On the well-posedness of initial-boundary value problems for higherorder linear hyperbolic equations with two independent variables," Differential Equations, vol. 39, no. 4, pp. 553-563, 2003.

[14] Z. Kamont, "Initial problems for hyperbolic functional differential equations with unbounded delay," Functional Differential Equations, vol. 8, no. 3-4, pp. 297-310, 2001.

[15] A. Nadolski, "Initial problems for quasi-linear hyperbolic functional differential systems with unbounded delay," Functional Differential Equations, vol. 9, no. 1-2, pp. 227-246, 2002.

[16] M. Grigolia, "On the existence and uniqueness of solutions of the Goursat problem for systems of functional partial differential equations of hyperbolic type," Memoirs on Differential Equations and Mathematical Physics, vol. 16, pp. 154-158, 1999.

[17] J. Šremr, "On the characteristic initial-value problem for linear partial functional-differential equations of hyperbolic type," Proceedings of the Edinburgh Mathematical Society. Series II, vol. 52, no. 1, pp. 241-262, 2009.

[18] A. Lomtatidze, S. Mukhigulashvili, and J. Šremr, "Nonnegative solutions of the characteristic initial value problem for linear partial functional-differential equations of hyperbolic type," Mathematical and Computer Modelling, vol. 47, no. 11-12, pp. 1292-1313, 2008.

[19] K. Deimling, "A Carathéodory theory for systems of integral equations," Annali di Matematica Pura ed Applicata, vol. 86, pp. 217-260, 1970.

[20] K. Deimling, "Absolutely continuous solutions of Cauchy's problem for $u_{x y}=f\left(x, y, u, u_{x}, u_{y}\right)$," Annali di Matematica Pura ed Applicata, vol. 89, pp. 381-391, 1971.

[21] K. Deimling, "Das Picard-Problem für $u_{x y}=f\left(x, y, u, u_{x}, u_{y}\right)$ unter Carathéodory-Voraussetzungen," Mathematische Zeitschrift, vol. 114, no. 4, pp. 303-312, 1970.

[22] C. Carathéodory, Vorlesungen über reelle Funktionen, Verlag und Druck Von B. G, Teubner, Berlin, Germany, 1918.

[23] J. Šremr, "Absolutely continuous functions of two variables in the sense of Carathéodory," Electronic Journal of Differential Equations, vol. 2010, no. 154, pp. 1-11, 2010.

[24] M. A. Krasnosel'skij, G. M. Vainikko, P. P. Zabrejko, Y. B. Rutickij, and V. Y. Stecenko, Approximate Solution of Operator Equations, Izdat. Nauka, Moscow, Russia, 1969.

[25] M. G. Krě̌n and M. A. Rutman, "Linear operators leaving invariant a cone in a Banach space," American Mathematical Society Translations, vol. 1950, no. 26, pp. 1-128, 1950. 


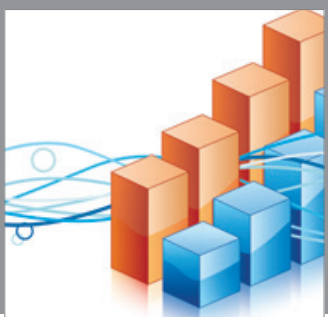

Advances in

Operations Research

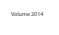

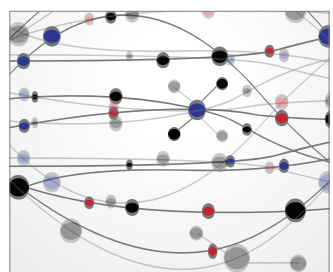

\section{The Scientific} World Journal
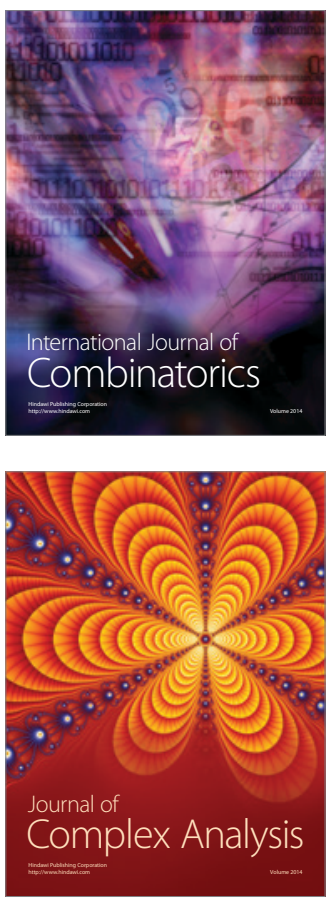

International Journal of

Mathematics and

Mathematical

Sciences
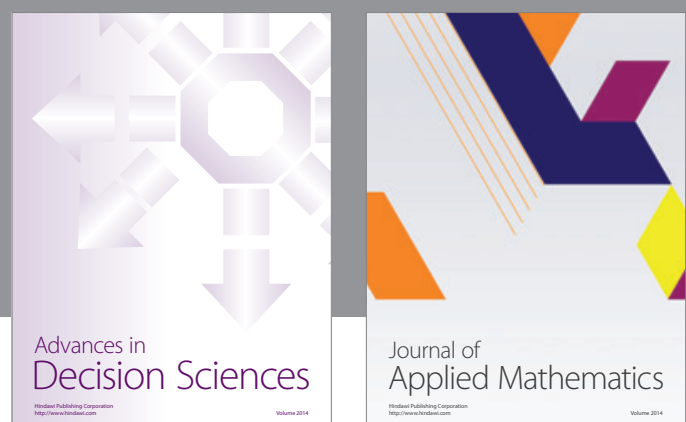

Journal of

Applied Mathematics
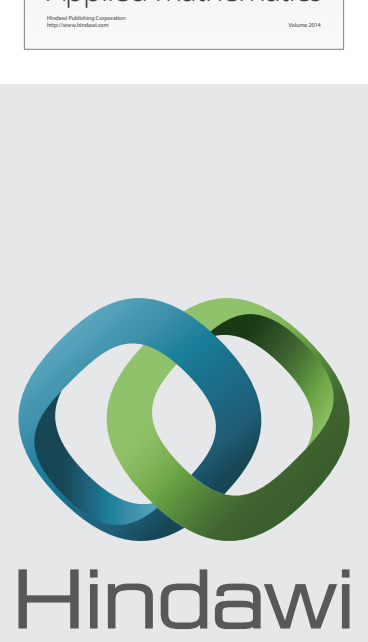

Submit your manuscripts at http://www.hindawi.com
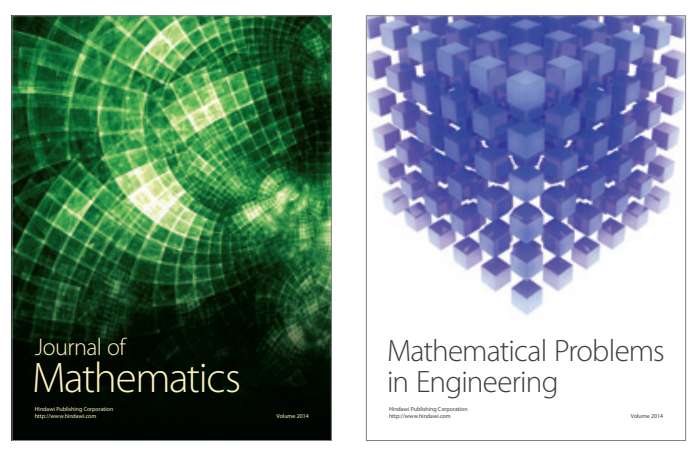

Mathematical Problems in Engineering
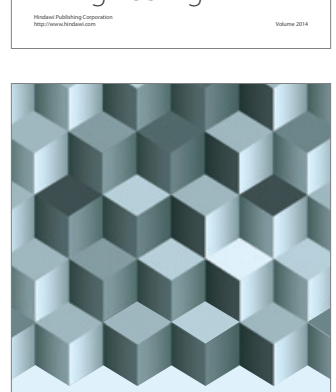

Journal of

Function Spaces
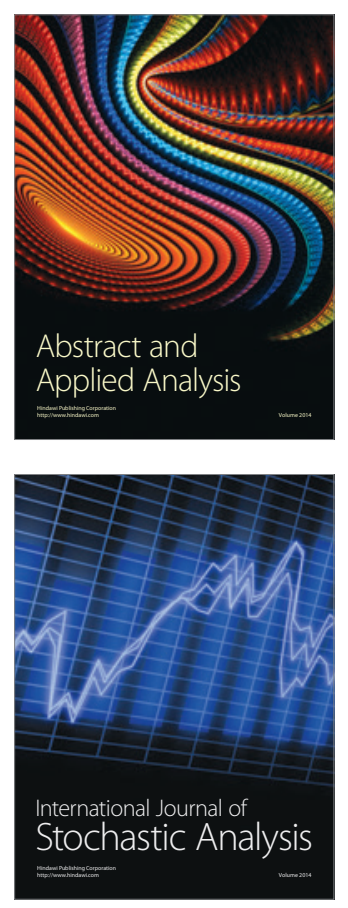

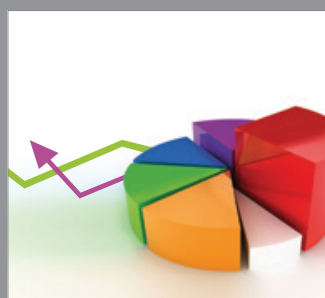

ournal of

Probability and Statistics

Promensencen
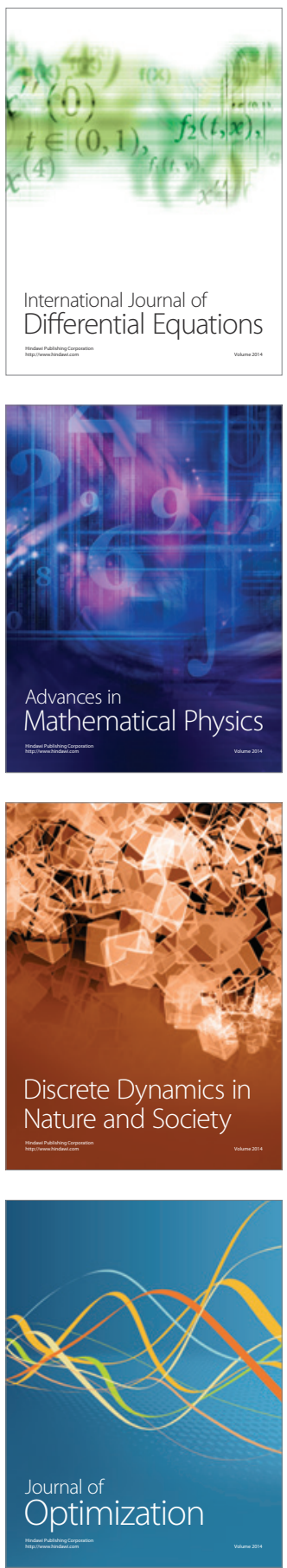